\title{
HOLOMORPHIC FAMILIES OF NON-EQUIVALENT EMBEDDINGS AND OF HOLOMORPHIC GROUP ACTIONS ON AFFINE SPACE
}

\author{
FRANK KUTZSCHEBAUCH AND SAM LODIN
}

\begin{abstract}
We construct holomorphic families of proper holomorphic embeddings of $\mathbb{C}^{k}$ into $\mathbb{C}^{n}(0<k<n-1)$, so that for any two different parameters in the family no holomorphic automorphism of $\mathbb{C}^{n}$ can map the image of the corresponding two embeddings onto each other. As an application to the study of the group of holomorphic automorphisms of $\mathbb{C}^{n}$ we derive the existence of families of holomorphic $\mathbb{C}^{*}$-actions on $\mathbb{C}^{n}(n \geq 5)$ so that different actions in the family are not conjugate. This result is surprising in view of the long standing Holomorphic Linearization Problem, which in particular asked whether there would be more than one conjugacy class of $\mathbb{C}^{*}$ actions on $\mathbb{C}^{n}$ (with prescribed linear part at a fixed point).
\end{abstract}

\section{IntroduCtion AND STATEMENT of the MAIN RESUlts.}

It is a famous theorem of Remmert that any Stein manifold of dimension $n$ admits a proper holomorphic embedding into affine $N$-space $\mathbb{C}^{N}$ of sufficiently high dimension $N$ [37]. Concerning this dimension, Eliashberg, Gromov [11] and Schürmann [40] proved that any Stein manifold of dimension $n>1$ can be embedded into $\mathbb{C}^{[3 n / 2]+1}$. A key ingredient in these results is the homotopy principle for holomorphic sections of elliptic submersions over Stein manifolds [26], [18], [15]. These dimensions are the smallest possible due to an example of Forster [12]. The optimal dimension for embeddings of Stein spaces can be found in Schürmann's paper [40].

2000 Mathematics Subject Classification. Primary 32M05, 32H02; Secondary 32Q28, 32Q40, 32Q45.

Key words and phrases. Complex Analysis; Proper Holomorphic Embeddings; Equivalent Embeddings; Eisenman Hyperbolicity; Complex Euclidean Spaces, Holomorphic Automorphisms, Group Actions, Oka-principle, Andersen-Lemperttheory.

The first author supported by Schweizerische Nationalfonds grant 200021$116165 / 1$. 
In this paper we do not investigate the question whether a given Stein space can be embedded into $\mathbb{C}^{N}$ for a given dimension $N$, but rather we investigate in how many ways this can be done in situations where at least one embedding exists. More precisely, we study the number of equivalence classes of proper holomorphic embeddings $\Phi: X \hookrightarrow \mathbb{C}^{n}$ with respect to the following equivalence relation:

Definition 1.1. Two embeddings $\Phi, \Psi: X \hookrightarrow \mathbb{C}^{n}$ are equivalent if there exist automorphisms $\varphi \in \operatorname{Aut}\left(\mathbb{C}^{n}\right)$ and $\psi \in \operatorname{Aut}(X)$ such that $\varphi \circ \Phi=$ $\Psi \circ \psi$.

In the algebraic case the question about the number of classes of equivalent embeddings $\mathbb{C}^{k} \hookrightarrow \mathbb{C}^{n}$ is well known and has been studied for a long time. The most famous result, due to Abhyankar and Moh [3], states that every polynomial embedding of $\mathbb{C}$ into $\mathbb{C}^{2}$ is equivalent to the standard embedding. The same is in general true for high codimension, Kaliman [31] proved that if $X$ is an affine algebraic variety and $n \geq$ $\max \{1+2 \operatorname{dim} X, \operatorname{dim} T X\}$ then all polynomial embeddings of $X$ into $\mathbb{C}^{n}$ are equivalent (by means of algebraic automorphisms). In the same paper Kaliman also proved that any polynomial embedding of $\mathbb{C}$ into $\mathbb{C}^{3}$ is holomorphically equivalent to the standard embedding. It is still an open question if this holds algebraically.

In the holomorphic case the situation is different. Rosay and Rudin [39] were the first to construct non-standard embeddings of $\mathbb{C}$ into $\mathbb{C}^{n}$, $n>2$, thus showing that the number of equivalence classes is at least two. Forstnerič, Globevnik and Rosay [16] showed that the result of Rosay and Rudin also holds for $n=2$. More generally, Forstnerič [14] showed that the number of equivalence classes of embeddings $\mathbb{C}^{k}$ into $\mathbb{C}^{n}$ is at least two for any $0<k<n$. Later, Derksen and the first author [9] proved that there are uncountably many non-equivalent embeddings of $\mathbb{C}$ into $\mathbb{C}^{n}$ for $n>1$. Their result heavily uses the fact that the holomorphic automorphism group of $\mathbb{C}$ is a Lie group, i.e., it is very small in comparison to the automorphism group of complex Euclidean spaces in dimensions greater than 1. Combining the ideas of that paper with the cancellation property for Eisenman hyperbolic spaces Borell and the first author then proved (see [6]) that the number of equivalence classes of proper holomorphic embeddings of $\mathbb{C}^{k}$ into $\mathbb{C}^{n}$ is uncountable for any $0<k<n$. The last two above mentioned results are proven by using the Cantor diagonal process and it remained still an unsolved challenging problem whether non-equivalent embeddings could occur in continuous or even holomorphic families.

Our first main result gives an affirmative answer. 
Theorem 1.1. Let $X$ be a complex space, which can be embedded into $\mathbb{C}^{n}$ and such that the group of holomorphic automorphisms $\operatorname{Aut}_{\text {hol }}(X)$ is a Lie group. Then there exist, for $k=n-1-\operatorname{dim} X$, a family of holomorphic embeddings of $X$ into $\mathbb{C}^{n}$ parametrized by $\mathbb{C}^{k}$, such that for different parameters $w_{1} \neq w_{2} \in \mathbb{C}^{k}$ the embeddings $\varphi_{w_{1}}, \varphi_{w_{2}}: X \hookrightarrow \mathbb{C}^{n}$ are non-equivalent.

Remark 1. Observe that for $k=0, \operatorname{dim} X=n-1$ the conclusion of the theorem is empty. In this situation it is still known that there are uncountably many equivalence classes of embeddings by the above mentioned results from [6].

We would like to emphasize that there is another (weaker) definition of equivalence - called Aut $\left(\mathbb{C}^{n}\right)$-equivalence - which is used by several authors, e.g., Buzzard, Forstnerič, Globevnik and Varolin. In these papers uncountability of certain equivalence classes of embeddings in this weaker sense is proved. Our main result is much stronger than these results. In our definition, two embeddings $\Phi, \Psi: X \hookrightarrow \mathbb{C}^{n}$ are equivalent if their images coincide modulo $\operatorname{Aut}\left(\mathbb{C}^{n}\right)$, i.e., if there is an automorphism $\varphi \in \operatorname{Aut}\left(\mathbb{C}^{n}\right)$ such that the images of $\varphi \circ \Phi$ and $\Psi$ coincide. In such a situation, the map $\Psi^{-1} \circ \varphi \circ \Phi$ is well defined and it is an automorphism of $X$. The weaker notion mentioned above demand that $\varphi \circ \Phi$ and $\Psi$ are equal as maps, i.e., it demands that $\Psi^{-1} \circ \varphi \circ \Phi$ is the identity on $X$. Our application to group actions would not work for the weaker definition.

Using the cancellation property for Eisenman hyperbolic spaces we can cross our situation with some affine space and we are able to conclude

Theorem 1.2. (see Corollary 4.1) There exist, for $k=n-l-1$, a family of holomorphic embeddings of $\mathbb{C}^{l}$ into $\mathbb{C}^{n}$ parametrized by $\mathbb{C}^{k}$, such that for different parameters $w_{1} \neq w_{2} \in \mathbb{C}^{k}$ the embeddings $\psi_{w_{1}}, \psi_{w_{2}}: \mathbb{C}^{l} \hookrightarrow \mathbb{C}^{n}$ are non-equivalent.

We also give an application of Theorem 1.1 to actions of compact (or equivalently complex reductive, see [34]) groups on $\mathbb{C}^{n}$. It was a long standing problem whether all holomorphic actions of such groups on affine space are linear after a change of variables (see for example the overview article [28]). The first counterexamples to that (Holomorphic Linearization) problem were constructed by Derksen and the first author in [8]. In the present paper we show that the method from there is holomorphic in a parameter and therefore applied to our parametrized situation leads to 
Theorem 1.3. For any $n \geq 5$ there is a holomorphic family of $\mathbb{C}^{*}$ actions on $\mathbb{C}^{n}$ parametrized by $\mathbb{C}^{n-4}$

$$
\mathbb{C}^{n-4} \times \mathbb{C}^{*} \times \mathbb{C}^{n} \rightarrow \mathbb{C}^{n} \quad(w, \theta, z) \mapsto \theta_{w}(z)
$$

so that for different parameters $w_{1} \neq w_{2} \in \mathbb{C}^{n-4}$ there is no equivariant isomorphism between the actions $\theta_{w_{1}}$ and $\theta_{w_{2}}$.

The linearization problem for holomorphic $\mathbb{C}^{*}$-actions on $\mathbb{C}^{n}$ is thus solved to the positive for $n=2$ by Suzuki [41] and still open for $n=3$. For $n=4$ there are uncountably many actions (non-linearizable ones among them) [9] and for $n \geq 5$ our result implies that there are families. Moreover there are families including a linear action as a single member of the family as our last main result shows

Theorem 1.4. For any $n \geq 5$ there is a holomorphic family of $\mathbb{C}^{*}$ actions on $\mathbb{C}^{n}$ parametrized by $\mathbb{C}$

$$
\mathbb{C} \times \mathbb{C}^{*} \times \mathbb{C}^{n} \rightarrow \mathbb{C}^{n} \quad(w, \theta, z) \mapsto \theta_{w}(z)
$$

so that for different parameters $w_{1} \neq w_{2} \in \mathbb{C}$ there is no equivariant isomorphism between the actions $\theta_{w_{1}}$ and $\theta_{w_{2}}$. Moreover the action $\theta_{0}$ is linear.

The paper is organized as follows. In section 2 we give all technical preparations for our (quite complicated) construction. The proofs will be given in the appendix. Section 3 contains the proof of Theorem 1.1. The next section 4 contains an addition to Theorem 1.1 which allows to deduce Theorem 1.2. Section 5 contains the application to group actions in particular the proofs of Theorems 1.3 and 1.4. Some concluding remarks are contained in section 6.

The results of the present paper have been partially announced in [35]. At that time the technical details had been extremely complicated and lengthy. Over the last years they have become much shorter and much more elegant so that the authors finally decided to publish the present complete version.

Part of the work was done during a stay of the first author at the Mittag-Leffler-Institute during the special program in Complex Analysis of Several Variables 2008. We would like to thank the Institute for hospitality and excellent working conditions.

\section{TEChNicAl PREPARATiOnS}

In this section we state the main lemmas needed to prove the main theorem. The proofs of theese lemmas can be found in section 7 .

For the benefit of the reader we give a list of notations mostly adhered to in this paper. 
By an automorphism of $\mathbb{C}^{n}$ depending on a parameter $w \in \mathbb{C}^{k}$ we mean an element of $\mathrm{Aut}_{h o l}^{k}\left(\mathbb{C}^{n}\right):=\left\{\psi \in \mathrm{Aut}_{h o l}\left(\mathbb{C}^{k+n}\right): \quad \psi(w, z)=\right.$ $\left.\left(w, \psi_{1}(w, z)\right)\right\}$, and approximations are understood to be uniform on compacts.

We will throughout the paper call a holomorphic map $\eta: \mathbb{C}^{k} \rightarrow \mathbb{C}^{n}$ a parametrized point $\eta(w)$ in $\mathbb{C}^{n}$.

- $\mathbb{B}_{i}$ is the open unit ball of $\mathbb{C}^{i}$. The closed ball is denoted $\overline{\mathbb{B}}_{i}$.

- $X$ a complex space of dimension $\operatorname{dim} X$.

- $\varphi_{0}=\iota: X \hookrightarrow \mathbb{C}^{n}$, where $\iota$ is the inclusion map.

- $\phi_{0}: \mathbb{C}^{k} \times X \rightarrow \mathbb{C}^{k} \times \mathbb{C}^{n}$ is given by $(w, x) \mapsto\left(w, \varphi_{0}(x)\right)$ for $w \in \mathbb{C}^{k}$.

- $\alpha_{n} \in \mathrm{Aut}_{\mathrm{hol}}^{\mathrm{k}}\left(\mathbb{C}^{\mathrm{n}}\right)$.

- $A_{n}=\alpha_{n} \circ \alpha_{n-1} \circ \ldots \circ \alpha_{1}$.

- $\phi_{n}=A_{n} \circ \phi_{0}=\alpha_{n} \circ \alpha_{n-1} \circ \ldots \circ \alpha_{1} \circ \phi_{0}: \mathbb{C}^{k} \times X \rightarrow \mathbb{C}^{k} \times \mathbb{C}^{n}$.

- $\pi_{2}$ the projection of $\mathbb{C}^{k} \times \mathbb{C}^{n}$ onto $\mathbb{C}^{n}$.

- $\phi=\lim _{n \rightarrow \infty} A_{n} \circ \phi_{0}(w, x)$.

- $\varphi_{n}=\pi_{2}\left(A_{n} \circ \phi_{0}\right)=\pi_{2}\left(\phi_{n}\right)$.

- $\varphi=\pi_{2}\left(A \circ \phi_{0}\right)$ (or $\varphi_{w}$ if $w$ is a fixed parameter value).

- $P_{2}: \mathbb{C}^{k} \times X \rightarrow X$ is given by $P_{2}(w, x)=x$.

- $\xi_{i}(w) \in \mathbb{C}^{n}$ interpolation points (osculation points) which vary with respect to $w \in \mathbb{C}^{k}$.

- $\eta_{i}$ the points of $X$ corresponding to the points $\xi_{i}(w)$ (preimage points of $\left.\xi_{i}(w)\right)$.

- $\mu$ induction variable. For every $\mu$ we define $\epsilon_{\mu}, R_{\mu}>0$ and finite subsets $\cup_{j=1}^{k(\mu)}\left\{a_{j}^{\mu}\right\}$ of $\partial(\mu+1) \mathbb{B}_{n}$ and $\cup_{j=1}^{k(\mu)}\left\{x_{j}^{\mu}\right\}$ of $X$ respectively.

2.1. Growth restrictions for holomorphic maps. In the construction of our families of non-equivalent embeddings we will use techniques of growth restrictions on entire maps from $\mathbb{C}^{n}$ to $\mathbb{C}^{n}$.

These growth restrictions are governed by the following lemma, Lemma 4.3 in 38 which we present with a simple additional conclusion, namely that one can avoid the nowhere dense set $Q$. The additional conclusion is obvious from the proof.

Lemma 2.1. Given real numbers $0<a_{1}<a_{2}, 0<r_{1}<r_{2}$ and $c>0$, let $\Gamma$ be the class of holomorphic mappings

$$
f=\left(f_{1}, \ldots, f_{k}\right): a_{2} \mathbb{B}_{n} \rightarrow r_{2} \mathbb{B}_{k}
$$

such that

$$
|f(0)| \leq \frac{1}{2} r_{1}
$$


and

at some point of $a_{1} \overline{\mathbb{B}}_{n}$.

$$
\left\|\frac{\partial\left(f_{1}, \ldots, f_{k}\right)}{\partial\left(z_{1}, \ldots, z_{k}\right)}\right\| \geq c
$$

Let $Q \subset \partial\left(r_{1} \mathbb{B}_{k}\right)$ be a set such that $\partial\left(r_{1} \mathbb{B}_{k}\right) \backslash Q$ is dense in $\partial\left(r_{1} \mathbb{B}_{k}\right)$. Then there is a finite set $E=E\left(a_{1}, a_{2}, r_{1}, r_{2}, c\right) \subset \partial\left(r_{1} \mathbb{B}_{k}\right) \backslash Q$ with the property that, if $f \in \Gamma$ and $f\left(a_{1} \mathbb{B}_{n}\right)$ intersects $\partial\left(r_{1} \mathbb{B}_{k}\right)$ then $f\left(a_{2} \mathbb{B}_{n}\right)$ intersects $E$.

The following technical detail is well known, we include for completeness, it is Lemma 5.4. in [14. It will be used frequently in the proof of Theorem 1.1.

Lemma 2.2. Let $K$ be a polynomially convex set in $\mathbb{C}^{n}$ and let $X$ be a closed analytic subvariety of $\mathbb{C}^{n}$. Moreover let $X_{0}$ be a compact holomorphically convex subset of $X$, such that $K \cap X_{0}$ is contained in the (relative) interior of $X_{0}$.

Then the set $K \cup X_{0}$ is polynomially convex.

We need to construct parametrized points with a certain property.

Proposition 2.1. Given natural numbers $k>0$ and $n \geq 2$ there is a number $m \in \mathbb{N}$ such there are $m$, pairwise different, parametrized points $\xi_{1}, \xi_{2}, \ldots, \xi_{m}: \mathbb{C}^{k} \rightarrow \mathbb{C}^{n}$ parametrized by a parameter $w \in \mathbb{C}^{k}$ with the following property:

For $w_{1} \neq w_{2}$ there is no affine automorphism $\alpha \in \operatorname{Aff}\left(\mathbb{C}^{n}\right)$ which maps the set of points $\left\{\xi_{1}\left(w_{1}\right), \xi_{2}\left(w_{1}\right), \ldots, \xi_{m}\left(w_{1}\right)\right\}$ onto the set of points $\left\{\xi_{1}\left(w_{2}\right), \xi_{2}\left(w_{2}\right), \ldots, \xi_{m}\left(w_{2}\right)\right\}$.

It is not difficult to see that the holomorphic (even the algebraic) automorphism group of $\mathbb{C}^{n}(n \geq 2)$ acts transitively on finite subsets of $\mathbb{C}^{n}$ with fixed cardinality, i.e. one can move $k$ distinct points $z_{1}, z_{2}, \ldots, z_{k} \in \mathbb{C}^{n}$ by an automorphism into some standard position, for example to the points $(1,0, \ldots, 0),(2,0, \ldots, 0), \ldots,(k, 0, \ldots, 0)$, (usually if the dimension is clear we will write $(k, 0))$. For this apply a generic linear change of coordinates so that afterwards all coordinates of the points $z_{1}, z_{2}, \ldots, z_{k}$ become different and then apply appropriate shears. The question whether the holomorphic automorphism group of $\mathbb{C}^{n}$ acts transitively on countable discrete subsets of $\mathbb{C}^{n}$ was answered to the negative by Rosay and Rudin in [38. They called the countable discrete subsets in the Aut ${ }_{h o l}\left(\mathbb{C}^{n}\right)$-orbit the "standard" countable discrete subset $e_{1} \mathbb{N}=\{(1,0),(2,0), \ldots,(k, 0), \ldots\}$ tame sets and proved the existence of non tame sets.

For our construction of families of embeddings we have to move finitely many points holomorphically depending on a parameter to some 
prescribed position by an automorphism which also depends holomorphically on the parameter.

Definition 2.1. Let $N$ be a natural number and $\zeta_{1}, \zeta_{2}, \ldots, \zeta_{N}: \mathbb{C}^{k} \rightarrow$ $\mathbb{C}^{n}$ be holomorphic maps such that for each fixed parameter $w \in \mathbb{C}^{k}$ the $N$ points $\zeta_{1}(w), \zeta_{2}(w), \ldots, \zeta_{N}(w)$ in $\mathbb{C}^{n}$ are different. We call them simultaneously standardizable, if there exists an automorphism $\psi \in$ $\mathrm{Aut}_{\text {hol }}^{k}\left(\mathbb{C}^{n}\right)$ holomorphically depending on the parameter $w$ with

$$
\psi\left(w, \zeta_{i}(w)\right)=(w,(i, 0)) \quad \text { for all } i=1,2, \ldots, N \quad \text { and for all } w \in \mathbb{C}^{k} \text {. }
$$

At the moment we are not able to decide under which condition on the parameter space any collection of parametrized points is simultaneously standardizable. We have the following partial result which is sufficient for our purposes.

Proposition 2.2. Let $\zeta_{1}, \zeta_{2}, \ldots, \zeta_{N}: \mathbb{C}^{k} \rightarrow \mathbb{C}^{n}, n \geq 2$, be holomorphic maps such that the points $\zeta_{1}(w), \zeta_{2}(w), \ldots, \zeta_{N}(w)$ in $\mathbb{C}^{n}$ are different for each $w \in \mathbb{C}^{k}$ and suppose $k<n-1$. Then the parametrized points $\zeta_{1}, \zeta_{2}, \ldots, \zeta_{N}$ are simultaneously standardizable.

2.2. Interpolation lemma. The following lemma is a key ingredient in the proof of our main theorem. It can be used to prove interpolation results for parametrized embeddings as this is well-known in the non parametrized version. For the non-parametrized case we refer to the papers [21], [33], 17], [14]. Another approach to interpolation is used in [36]. We do not prove parametrized interpolation theorems for countable sets in this paper, we just use the lemma as a tool in the proof of our main Theorem 1.1.

Therefore before we formulate the lemma we need to introduce some notation:

Let $\phi: \mathbb{C}^{k} \times X \hookrightarrow \mathbb{C}^{k} \times \mathbb{C}^{n}$ be a (parametrized) embedding of a complex space $X$ into $\mathbb{C}^{n}$, i.e., an embedding of the form $\phi(w, x)=$ $(w, \tilde{\phi}(w, x))$.

$X_{R}$ is a holomorphically convex compact subset of $X$.

$\bar{\Delta}$ is a ball (of any radius) in $\mathbb{C}^{k} . \overline{\mathbb{B}}$ is a ball (of any radius) in $\mathbb{C}^{n}$.

We assume that $\phi_{w}^{-1}(\overline{\mathbb{B}}) \subset X_{R}$ for all $w \in \bar{\Delta}$, which implies that $K=(\bar{\Delta} \times \overline{\mathbb{B}}) \cup \phi\left(\bar{\Delta} \times \bar{X}_{R}\right)$ is a polynomially convex subset of $\mathbb{C}^{k} \times \mathbb{C}^{n}$ (Lemma 2.2 above).

Furthermore we assume the dimension condition

$$
\operatorname{dim} X+k<n \text {. }
$$

Lemma 2.3. Let $b_{1}(w), b_{2}(w), \ldots, b_{N}(w)$ be $N$ parametrized points contained in $K=(\bar{\Delta} \times \overline{\mathbb{B}}) \cup \phi\left(\bar{\Delta} \times \bar{X}_{R}\right)$. Assume that $(w, p(w))$ 
and $(w, q(w))$ are parametrized points in $\mathbb{C}^{k} \times \mathbb{C}^{n} \backslash K$ and let $s$ be some positive integer. Then for each $\epsilon>0$ there exists an automorphism $\alpha \in$ Aut $_{\mathrm{hol}}^{\mathrm{k}}\left(\mathbb{C}^{\mathrm{n}}\right)$ such that $|\alpha(w, z)-(w, z)| \leq \epsilon$ for every $(w, z) \in K, \alpha\left(w, b_{i}(w)\right)=\left(w, b_{i}(w)\right)$ of order $s$ for every $w \in \mathbb{C}^{k}$ and $\alpha(w, p(w))=(w, q(w))$ for every $w \in \mathbb{C}^{k}$.

2.3. Osculation lemma. The conclusion of the growth restrictions will be that only affine automorphisms could map the images of different embeddings from the family onto each other. To exclude these affine automorphisms we will have a finite number of points "marked" in each embedding of our family. The marking is in such a way that by affine automorphisms the sets of marked points have to be mapped onto each other. The marking will be achieved by letting the embeddings osculate of higher order exactly at these points.

Definition 2.2. Let $l \geq 2$ be a natural number. We say that a submanifold $M$ of $\mathbb{C}^{n}$ osculates of order $l$ at $x \in M$ if $M$ has contact order $l$ with the tangent space $T_{x} M \subset \mathbb{C}^{n}$ in $x \in M$.

In local coordinates osculating can be interpreted as follows:

Let $\zeta: U\left(\subset \mathbb{C}^{m}\right) \rightarrow M$ be a holomorphic coordinate system for the $m$ dimensional manifold $M$ at $x, \zeta(0)=x$. Then $M$ osculates of order $l$ at $x$ if and only if $\left.\frac{\partial}{\partial w^{\alpha}}\right|_{w=0} \zeta \in T_{x} M$ for every multiindex $\alpha=\left(\alpha_{1}, \alpha_{2}, \ldots, \alpha_{m}\right)$ with $2 \leq|\alpha| \leq l$.

The property to osculate is preserved by affine coordinate changes on $\mathbb{C}^{n}$, i.e. if $\psi: \mathbb{C}^{n} \rightarrow \mathbb{C}^{n}$ is an affine automorphism of $\mathbb{C}^{n}$ then the submanifold $M \subset \mathbb{C}^{n}$ osculates of order $l$ at $x \in M$ if and only if the submanifold $\psi(M)$ osculates of order $l$ at $\psi(x) \in \psi(M)$.

Remark 2. (1) The property not to osculate of order $l$ at any point is generic for $l \geq 2$ if not $\operatorname{dim} M=1$ and $n=2$. In the later case it is generic for $l \geq 3$.

(2) In the proof of Theorem 1.1 the role of $M$ will be played by the smooth part $X \backslash \operatorname{Sing}(X)$ of the space we want to embed.

By a manifold $M_{\mathbb{C}^{k}}$ in $\mathbb{C}^{n}$ parametrized by $w \in \mathbb{C}^{k}$ we mean the image (in $\mathbb{C}^{k} \times \mathbb{C}^{n}$ ) of a proper holomorphic embedding $\Phi: \mathbb{C}^{k} \times M \rightarrow \mathbb{C}^{k} \times \mathbb{C}^{n}$ which is of form $(w, m) \mapsto(w, \phi(w, m))$. By $M(w)$ we denote the image $\Phi(\{w\} \times M) \subset \mathbb{C}^{n}$.

Lemma 2.4. Let $M_{\mathbb{C}^{k}}$ be a manifold in $\mathbb{C}^{n}$ parametrized by $w \in \mathbb{C}^{k}$ and assume that $m=\operatorname{dim} M<n$. Let $\xi_{1}(w), \ldots, \xi_{t}(w)$ be simultaneously standardizable parametrized points such that $\xi_{i}(w) \in M(w)$ for $i=1, \ldots, t$. Then for $l \geq 2 \in \mathbb{N}$ there exists an automorphism $\kappa \in \mathrm{Aut}_{\mathrm{hol}}^{\mathrm{k}}\left(\mathbb{C}^{\mathrm{n}}\right)$ such that $\kappa(M(w))$ osculates of order $l$ in $\xi_{i}(w)$ for $i=1, \ldots, t$ and all $w \in \mathbb{C}^{k}$. 
2.4. Non-osculation lemma. To keep the osculation of order $l$ in $\xi_{i}(w)$ and make sure that the embedding do not osculates of order $l$ at other points, we use the following lemma.

Lemma 2.5. Let $M_{\mathbb{C}^{k}}$ be a manifold in $\mathbb{C}^{n}$ parametrized by $w \in \mathbb{C}^{k}$ and denote $m=\operatorname{dim} M$. Also let

a) $K_{M}$ be a compact subset of $\mathbb{C}^{k} \times M_{\mathbb{C}^{k}}$.

b) $K$ be a compact subset of $\mathbb{C}^{k} \times \mathbb{C}^{n}$.

c) $a_{1}, a_{2}, \ldots, a_{r}$ be finitely many points in $K_{M}$.

d) $b_{1}(w), b_{2}(w), \ldots, b_{q}(w)$ be finitely many parametrized points in $\mathbb{C}^{k} \times$ $M(w) \backslash K_{M}$, (these are the points where we want to keep the osculation order $l)$.

e) $l \geq 2$ be a natural number. If $m=1$ and $n=2$ let $l \geq 3$.

f) $\epsilon>0$ be a real number.

Then there exists an automorphism $\psi \in \mathrm{Aut}_{\mathrm{hol}}^{\mathrm{k}}\left(\mathbb{C}^{\mathrm{n}}\right)$ such that

1. $\psi\left(a_{i}\right)=a_{i}$ for every $i=1,2, \ldots, r$.

2. $\psi_{w}(z)=z+O\left(\left|z-b_{i}(w)\right|^{l+1}\right)$ as $z \rightarrow b_{i}$ for every $i=1,2, \ldots, q$ and every $w \in \mathbb{C}^{k}$.

3. $\left|\psi_{w}(z)-z\right|+\left|\psi_{w}^{-1}(z)-z\right|<\epsilon$ for every $(w, z) \in K$

4. There is no point $x \in K_{M}$ such that $\psi(M(w))$ osculates of order $l$ in $\psi(x)$.

\section{Proof of MAIN THEOREM.}

To be precise let us define the notion used in the formulation of Theorem 1.1

Definition 3.1. Let $X, Y$ be complex spaces, $Z$ a complex (resp. topological) space. A holomorphic (resp. continuous) map

$$
\Phi: Z \times X \rightarrow Y
$$

is called a holomorphic (resp. continuous) family of holomorphic embeddings of $X$ into $Y$ parametrized by (a parameter) in $Z$ if for each point $z$ in the parameter space $Z$ the map $\Phi_{z}: X \rightarrow Y, \quad x \mapsto \Phi(z, x)$ is a proper holomorphic embedding.

In the proof we are working with families of embeddings of some complex space $X$ into $\mathbb{C}^{n}$ parametrized by $\mathbb{C}^{k}$ which come from the following construction.

Remark 3. If a holomorphic map

$$
\phi: \mathbb{C}^{k} \times X \rightarrow \mathbb{C}^{k} \times \mathbb{C}^{n}
$$

of the form 


$$
\phi(w, x)=(w, \tilde{\phi}(w, x)), \quad w \in \mathbb{C}^{k}, x \in X
$$

is a proper holomorphic embedding of $\mathbb{C}^{k} \times X$ into $\mathbb{C}^{k} \times \mathbb{C}^{n}$ then the map

$$
\tilde{\phi}: \mathbb{C}^{k} \times X \rightarrow \mathbb{C}^{n}
$$

(where $\tilde{\phi}$ is defined by (2)) is a family of holomorphic embeddings of $X$ into $\mathbb{C}^{n}$ parametrized by $\mathbb{C}^{k}$. Note that the contrary does not hold, i.e., if

$$
\tilde{\phi}: \mathbb{C}^{k} \times X \rightarrow \mathbb{C}^{n}
$$

is a family of holomorphic embeddings of $X$ into $\mathbb{C}^{n}$ parametrized by $\mathbb{C}^{k}$, the corresponding map

$$
\phi: \mathbb{C}^{k} \times X \rightarrow \mathbb{C}^{k} \times \mathbb{C}^{n}
$$

defined by

$$
\phi(w, x)=(w, \tilde{\phi}(w, x)), \quad w \in \mathbb{C}^{k}, x \in X
$$

may fail to be an embedding (see the example below).

Also note the following fact which we will use in our construction : If $\alpha \in \mathrm{Aut}_{h o l}\left(\mathbb{C}^{k} \times \mathbb{C}^{n}\right)$ is of the form $\alpha(w, z)=(w, \tilde{\alpha}(w, z))$, i.e. $\alpha \in$ Aut $_{h o l}^{k}\left(\mathbb{C}^{n}\right)$, then $\alpha \circ \phi$ is again an embedding of the form (2), hence its "second coordinate" $\pi_{2} \circ(\alpha \circ \phi): \mathbb{C}^{k} \times X \rightarrow \mathbb{C}^{n}$ is a family of holomorphic embeddings (where $\pi_{2}: \mathbb{C}^{k} \times \mathbb{C}^{n} \rightarrow \mathbb{C}^{n}$ is defined by $\left.(w, v) \mapsto v, \quad w \in \mathbb{C}^{k}, v \in \mathbb{C}^{n}\right)$.

Example 1. If

$$
\tilde{\phi}: \mathbb{C}^{k} \times X \rightarrow \mathbb{C}^{n}
$$

is a holomorphic family of holomorphic embeddings of $X$ into $\mathbb{C}^{n}$ parametrized by $\mathbb{C}^{k}$ then it is straightforward to prove that the map

$$
\phi: \mathbb{C}^{k} \times X \rightarrow \mathbb{C}^{k} \times \mathbb{C}^{n}
$$

defined by

$$
\phi(w, x)=(w, \tilde{\phi}(w, x)), \quad w \in \mathbb{C}^{k}, x \in X
$$

is holomorphic, injective and immersive. On the other hand properness may fail, as the following example shows: We are going to define a holomorphic family of embeddings of $\mathbb{C}$ into $\mathbb{C}^{2}$ parametrized by $\mathbb{C}$. Define $f: \mathbb{C} \times \mathbb{C} \rightarrow \mathbb{C}^{2}$ by $f(y, x)=\left(x+y \cdot x^{2}, y \cdot x\right)$. For each fixed point $y$ in the parameter space $\mathbb{C}$ we are given a proper, injective, immersive, holomorphic map from $\mathbb{C}$ into $\mathbb{C}^{2}$ (for $y \neq 0$ the second coordinate by itself gives already such an embedding, and for $y=0$ the first coordinate is such an imbedding).

On the other hand the map $\mathbb{C} \times \mathbb{C} \rightarrow \mathbb{C} \times \mathbb{C}^{2}$ defined by $(y, x) \mapsto$ $\left(y, x+y \cdot x^{2}, y \cdot x\right)$ is not proper. Indeed the sequence $\left(x_{n}, y_{n}\right)$ defined by 
$x_{n}=n$ and $y_{n}=\frac{1-n}{n^{2}}$ leaves any compact subset of the definition space but is mapped onto the sequence $\left(\frac{1-n}{n^{2}}, 1, \frac{1-n}{n}\right)$ which converges to the point $(0,1,-1)$ in the target space (which is not in the image, in fact the image is not closed it is the hypersurface $\left\{(a, b, c) \in \mathbb{C}^{3}: a \cdot b=c(c+1)\right\}$ except the line $\{c=-1, a=0\})$.

We would like to emphasize that we will prove a slightly stronger statement than just holomorphic families of embeddings. Our families are always such that the map $\Phi: \mathbb{C}^{k} \times X \rightarrow \mathbb{C}^{k} \times \mathbb{C}^{n}$ is a proper holomorphic embedding which we will denote by using the symbol $\hookrightarrow$, i.e. $\Phi: \mathbb{C}^{k} \times X \hookrightarrow \mathbb{C}^{k} \times \mathbb{C}^{n}$ for our families. This subtle point plays a role in the last section, since if $\Phi\left(\mathbb{C}^{k} \times X\right)$ is not a closed submanifold in $\mathbb{C}^{k} \times \mathbb{C}^{n}$ the construction of pseudo-affine modification does not work.

Remark 4. In the proof of Theorem 1.1 we use the property that the group of holomorphic automorphisms of $X$ can be exhausted by a sequence of compact subsets (in c.-o. topology). A Lie group (with possibly countably many components) can be exhausted by a sequence of compact subsets. On the other hand, if a locally compact topological group acts effectively on a manifold it is a Lie group [7]. Since the group of holomorphic automorphisms of a Stein space $X$ acts effectively on the smooth part $\tilde{X}=X \backslash \operatorname{Sing}(X)$, this implies that $\operatorname{Aut}(X)$ is a Lie group if and only if it can be exhausted by compacts.

Proof. (of Theorem 1.1)

By assumption the complex space $X$ embeds into $\mathbb{C}^{n}$, say $\varphi_{0}: X \hookrightarrow$ $\mathbb{C}^{n}$. We start with the trivial family of embeddings of $X$ into $\mathbb{C}^{n}$, $\phi_{0}: \mathbb{C}^{k} \times X \rightarrow \mathbb{C}^{k} \times \mathbb{C}^{n}$ given by $(w, x) \mapsto\left(w, \varphi_{0}(x)\right)$.

We will construct automorphisms $\alpha_{n} \in \mathrm{Aut}_{h o l}^{k}\left(\mathbb{C}^{n}\right)$ recursively. Let $A_{n}=\alpha_{n} \circ \alpha_{n-1} \circ \ldots \circ \alpha_{1}$. We further arrange $\alpha_{n} \in \mathrm{Aut}_{h o l}^{k}\left(\mathbb{C}^{n}\right)$ inductively such that

$$
A(w, z)=\lim _{n \rightarrow \infty} A_{n}(w, z)=\left(w, \lim _{n \rightarrow \infty} \tilde{A}_{n}(w, z)\right)
$$

exist uniformly on compacts for some open neighborhood $\Omega \subset \mathbb{C}^{k} \times \mathbb{C}^{n}$ containing $\mathbb{C}^{k} \times X$ and such that the mapping $A: \Omega \rightarrow \mathbb{C}^{k} \times \mathbb{C}^{n}$ given by $(w, z) \mapsto\left(w, \tilde{A}_{n}(w, z)\right)$ defines a biholomorphic mapping on $\mathbb{C}^{k} \times \mathbb{C}^{n}$. The existence of the limit follows from Proposition 4.1 and 4.2 in [14.

Now let $\phi_{n}=\alpha_{n} \circ \alpha_{n-1} \circ \ldots \circ \alpha_{1} \circ \phi_{0}: \mathbb{C}^{k} \times X \rightarrow \mathbb{C}^{k} \times \mathbb{C}^{n}$. Also define $\pi_{2}$ as the projection of $\mathbb{C}^{k} \times \mathbb{C}^{n}$ to $\mathbb{C}^{n}$.

The family of holomorphic embeddings will then be given by the second coordinate of $A \circ \phi_{0}=\lim _{n \rightarrow \infty} \phi_{n}$, i.e. $\varphi=\pi_{2}\left(A \circ \phi_{0}\right)$. 
It follows that $\phi=\lim _{n \rightarrow \infty} A_{n} \circ \phi_{0}=A \circ \phi_{0}$ is a proper holomorphic embedding of $\mathbb{C}^{k} \times X$ into $\mathbb{C}^{k} \times \mathbb{C}^{n}$, which gives that $\varphi=\pi_{2}\left(A \circ \phi_{0}\right)$ is a family of holomorphic embeddings of $X$ into $\mathbb{C}^{n}$ parametrized by $\mathbb{C}^{k}$.

In order to make the embeddings $\phi_{w}$ different for different parameters, we will choose them such that no affine automorphism, can map the image of one of these embeddings onto another. At the same time we make sure, using growth conditions, that the only way to map the image of one embedding onto another is by an affine mapping. This ensures that the embeddings will be non-equivalent for different parameters $w$.

So the construction in short is:

a) Choose sufficiently many points $\xi_{1}(w), \ldots, \xi_{m}(w)$ in correct positions such that no affine automorphism can map the points $\left\{\xi_{i}\left(w_{1}\right)\right\}$ into the points $\left\{\xi_{i}\left(w_{2}\right)\right\}$ for $w_{1} \neq w_{2}$. These points will be chosen differently for each parameter (however holomorphically depending on the parameter).

b) Embed the space $X$ through these points.

c) In order to single out these points we make sure that our embeddings osculate at these points of a certain order $l$, and osculates of order less than $l$ at all other points.

d) Divide the rest of $\mathbb{C}^{n}$ into concentric shells with increasing radii. In each shell we choose inductively points through which we later will embed the space $X$. These points and their preimages in $X$ are inductively chosen in such a way that we get some growth conditions on the embedding.

e) Embed $X$ inductively through all the points with careful chosen preimage points. In each step of the inductive process $X$ will be embedded through the (finitely many) points contained in one shell, keeping the points from previous shells contained in the image of $X$.

f) We then show, using the growth conditions introduced in $d$ ), that for two embeddings to be equivalent for different parameters they have to differ by an affine automorphism.

g) The condition in a) shows that there is no such affine automorphism. Consequently the embeddings are different for all parameters.

The first automorphism $\alpha_{1}$ will take care of that for different parameters $w_{1} \neq w_{2} \in \mathbb{C}^{k}$ there is no affine automorphism $\beta \in \operatorname{Aff}\left(\mathbb{C}^{n}\right)$ mapping the image of $\varphi_{w_{1}}(X)$ onto the image of $\varphi_{w_{2}}(X)$. For this first use Proposition 2.1 to get $m$ points $\xi_{1}, \ldots \xi_{m}: \mathbb{C}^{k} \rightarrow \mathbb{C}^{n}$ parametrized by an parameter. Then choose $m$ points, $\eta_{1}, \ldots, \eta_{m}$ in the smooth part $\tilde{X}$ of $X$ or more exactly of $\phi_{0}(X) \subset \mathbb{C}^{n}$. After that use Lemma 2.3 to 
find an automorphism $\gamma \in \operatorname{Aut}_{h o l}^{k}\left(\mathbb{C}^{n}\right)$ such that $\gamma\left(w, \eta_{i}\right)=\left(w, \xi_{i}(w)\right)$ for every $w \in \mathbb{C}^{k}$.

Fix a natural number $l \geq 2$ such that not osculating of order $l$, at any point, is a generic property for a submanifold of $\operatorname{dim} X$ in $\mathbb{C}^{n}$ (see Remark 2). Using Lemma 2.4 and Lemma 2.5 we get an automorphism $\delta \in \operatorname{Aut}_{h o l}^{k}\left(\mathbb{C}^{n}\right)$ with $\delta\left(w, \xi_{i}(w)\right)=\left(w, \xi_{i}(w)\right)$ for every $w \in \mathbb{C}^{k}$ that prescribes the higher derivatives of $\delta$ in the $\mathbb{C}^{n}$-direction such that for all $w \in \mathbb{C}^{k}$ the subvariety $\delta\left(\gamma\left(w, \varphi_{0}(X)\right)\right)$ of $\mathbb{C}^{n}$ will be tangent of order $l$ at the points $\xi_{i}(w)$.

The automorphism $\alpha_{1} \in \mathrm{Aut}_{h o l}^{k}\left(\mathbb{C}^{n}\right)$ is now given by $\alpha_{1}=\delta \circ \gamma$. We also note that with $\alpha_{1}$ we have the osculation order in the points we want, so in the future we only have to make sure we do not destroy the osculation order in $\xi_{i}(w)$ but destroy it everywhere else.

We now choose an exhaustion of the space $T=\operatorname{Aut}(X)$ with compact sets $T_{i}$ such that

$$
T=\cup_{i=1}^{\infty} T_{i} \text { and } T_{i} \subset \stackrel{\circ}{T}_{i+1} .
$$

Further let $\rho: X \rightarrow \mathbb{R}^{\geq 0}$ be a continuous exhaustion function of $X$, so $X_{r}:=\rho^{-1}([0, r])$ is a compact subset of $X$ for every $r \geq 0$. $\left(X_{r}=\right.$ $\{x \in X: \rho(x)<r\} \subset \subset X$ for every $r$, for example $\rho(x)=\|\iota(x)\|^{2}$ will work.)

Denote the unit ball in $\mathbb{C}^{n}$ by $\mathbb{B}_{n}=\left\{z \in \mathbb{C}^{n}:\|z\|<1\right\}$ and $\mathbb{B}_{k}=\left\{w \in \mathbb{C}^{k}:\|w\|<1\right\}$. Choose a sequence of relatively open neighborhoods $U_{i}, i=1,2,3, \ldots$ of the set $\eta=\cup_{i=1}^{m}\left\{\eta_{i}\right\}$ in $X$ with $\cap_{i=1}^{\infty} U_{i}=\eta$ and $\bar{U}_{i+1} \subset \stackrel{\circ}{U}_{i}$.

Remember that the points $\eta_{i}$ in $X$ are the preimages of the points in $\mathbb{C}^{n}$ at which the varieties $\varphi(w, X)$ have osculation of order $l$, i.e. the points $\xi_{i}(w)$, for every $w \in \mathbb{C}^{k}$.

Now we inductively, for $\mu=1,2,3, \ldots$, define real numbers $\epsilon_{\mu}, R_{\mu}>$ 0 , finite subsets $\cup_{j=1}^{k(\mu)}\left\{a_{j}^{\mu}\right\}$ of $\partial(\mu+1) \mathbb{B}_{n}$ and finite subsets $\cup_{j=1}^{k(\mu)}\left\{x_{j}^{\mu}\right\}$ of $X$ with the same cardinality $k(\mu)$, and automorphisms $\alpha_{\mu} \in \operatorname{Aut}_{h o l}^{k}\left(\mathbb{C}^{n}\right)$ of $\mathbb{C}^{n}$ parametrized by $w \in \mathbb{C}^{k}$. When choosing the subsets $\cup_{j=1}^{k(\mu)}\left\{a_{j}^{\mu}\right\}$ and $\cup_{j=1}^{k(\mu)}\left\{x_{j}^{\mu}\right\}$ it is important to remember that since we are going to embed the point $x_{j}^{\mu}$ of $X$ through $a_{j}^{\mu}$, we have to choose the points $a_{j}^{\mu}$ such that $\cup_{j=1}^{k(\mu)}\left\{a_{j}^{\mu}\right\} \cap \pi_{2}\left(\phi_{\mu}\left(\mathbb{C}^{k} \times X\right)\right)=\emptyset$. This is possible by Lemma 2.1 due to the dimension of $X$, since $\pi_{2}\left(\phi_{\mu}\left(\mathbb{C}^{k} \times X\right)\right) \cap \partial(\mu+1) \mathbb{B}_{n}$ has measure zero in $\partial(\mu+1) \mathbb{B}_{n}$.

Start by letting $\epsilon_{1}=1, R_{1}=1, k(1)=m$ and $\alpha_{1}$ be as constructed earlier. For $\mu \geq 2$ we construct these entities such that the following properties are satisfied: 
$1_{\mu} .0<\epsilon_{\mu}<\frac{\epsilon_{\mu-1}}{3}$

$2_{\mu}$. If $F: \mathbb{B}_{n} \rightarrow(\mu+2) \mathbb{B}_{n} \backslash \cup_{j=1}^{k(\mu)}\left\{a_{j}^{\mu}\right\}$ is a holomorphic mapping with $\|F(0)\| \leq \frac{\mu+1}{2}$ and $|J F(0)| \geq 1$ then $F\left(\left(1-\frac{\epsilon_{\mu}}{2}\right) \mathbb{B}_{n}\right) \subset(\mu+1) \mathbb{B}_{n}$.

$3_{\mu} . \phi_{\mu}\left(w, x_{j}^{\mu}\right)=\alpha_{\mu} \circ \phi_{\mu-1}\left(w, x_{j}^{\mu}\right)=\left(w, a_{j}^{\mu}\right)$ and $\rho\left(x_{j}^{\mu}\right)>\max \{\rho(t(x))$ : $\left.t \in T_{\mu}, x \in P_{2}\left(\phi_{\mu-1}^{-1}\left(\mu \overline{\mathbb{B}}_{k} \times \mu \overline{\mathbb{B}}_{n}\right)\right)\right\}$ where $P_{2}: \mathbb{C}^{k} \times X \rightarrow X$ is given by $P_{2}(w, x)=x$.

$4_{\mu} \cdot\left\|\alpha_{\mu} \circ \phi_{\mu-1}(w, x)-\phi_{\mu-1}(w, x)\right\|=\left\|\phi_{\mu}(w, x)-\phi_{\mu-1}(w, x)\right\| \leq \epsilon_{\mu}$ for every $x \in X_{R_{\mu-1}}$ and for every $w \in(\mu-1) \overline{\mathbb{B}}_{k}$.

$5_{\mu} .\left\|\alpha_{\mu}(w, z)-(w, z)\right\| \leq \epsilon_{\mu}$ for $(w, z) \in \mu \overline{\mathbb{B}}_{k} \times \mu \overline{\mathbb{B}}$

Remark 5 . Motivated by $4_{\mu}$ and $5_{\mu}$ we define the compact set

$$
K_{\mu}=\left(\mu \overline{\mathbb{B}}_{k} \times \mu \overline{\mathbb{B}}_{n}\right) \cup \phi_{\mu-1}\left((\mu-1) \overline{\mathbb{B}}_{k} \times \bar{X}_{R_{\mu-1}}\right) .
$$

$6_{\mu} \cdot \alpha_{\mu} \circ \phi_{\mu-1}\left(w, x_{j}^{l}\right)=\left(w, a_{j}^{l}\right)$ for every $w \in \mathbb{C}^{k}$ and for every $l<\mu$, $j=1,2, \ldots, k(l)$.

$7_{\mu} \cdot \alpha_{\mu}(w, z)=(w, z)+O\left(\left|z-\xi_{i}(w)\right|^{l+1}\right)$ as $z \rightarrow \xi_{i}(w)$.

$8_{\mu}$. For fix $w \in \mu \overline{\mathbb{B}}_{k}$ the submanifold $\pi_{2}\left(\phi_{\mu}(w, \tilde{X})\right)$ of $\mathbb{C}^{n}$ do not osculate of order $l$ in any point $\pi_{2}\left(\phi_{\mu}(w, x)\right)$ with $x \in\left(X_{R_{\mu-1}} \cap \tilde{X}\right) \backslash U_{\mu}$. Here $\tilde{X}$ is the union of all components of $X$ which are smooth and of maximal dimension.

$9_{\mu}$. $\left\|\phi_{\mu}(w, x)\right\| \geq \mu+1$ for every $x \in X \backslash X_{R_{\mu}}$ and for every $w \in \mu \overline{\mathbb{B}}_{k}$. $10_{\mu} \cdot R_{\mu}>R_{\mu-1}+1$.

We will now confirm that such a construction is possible.

For step 2 of the induction we choose, in the following order,

(1) $\epsilon_{2}<\frac{\epsilon_{1}}{3}$

(2) $\cup_{j=1}^{k(2)}\left\{a_{j}^{2}\right\}$ a finite subset of $\partial\left(3 \mathbb{B}_{n}\right) \subset \mathbb{C}^{n}$ which does not intersect the image $\pi_{2}\left(\phi_{1}\left(\mathbb{C}^{k} \times X\right)\right)$ and satisfies $2_{2}$. This is possible by Lemma 2.1, namely $\operatorname{dim} X+k<n$ makes it possible to choose the points $\left\{a_{j}^{2}\right\}$ outside $\pi_{2}\left(\phi_{1}\left(\mathbb{C}^{k} \times X\right)\right)$ (which has measure zero by Sards theorem).

(3) $\cup_{j=1}^{k(2)}\left\{x_{j}^{2}\right\}$ a finite subset of $X$ such that $\rho\left(x_{j}^{2}\right)>\max \{\rho(t(x)): t \in$ $\left.T_{2}, x \in P_{2}\left(\phi_{1}^{-1}\left(2 \overline{\mathbb{B}}_{k} \times 2 \overline{\mathbb{B}}_{n}\right)\right)\right\}$. Property $3_{2}$ will then be fulfilled. We shall also choose $x_{j}^{2}$ such that $\left(w, x_{j}^{2}\right) \notin \hat{K}_{2}$ for every $j$, where $K_{2}=\left(2 \overline{\mathbb{B}}_{k} \times 2 \overline{\mathbb{B}}_{n}\right) \cup \phi_{1}\left(1 \overline{\mathbb{B}}_{k} \times \bar{X}_{R_{1}}\right)$ is a compact subset of $\mathbb{C}^{k} \times \mathbb{C}^{n}$. As $\phi_{1}$ is a proper holomorphic embedding, Lemma 2.2 gives that $\hat{K}_{2}$ is contained in $\left(2 \overline{\mathbb{B}}_{k} \times 2 \overline{\mathbb{B}}_{n}\right) \cup \phi_{1}\left(1 \overline{\mathbb{B}}_{k} \times \bar{X}\right)$, in particular the points $\left(w, a_{j}^{2}\right)$ will not intersect the set $\hat{K}_{2}$.

By our dimension assumptions $(k<n-1)$ the parametrized points $\left\{\left(w, a_{j}^{2}\right),\left(w, x_{j}^{2}\right)\right\}$ are simultaneously standardizable (Corollary 2.2). We 
will now use Lemma $2.3 k(2)$ times to find an automorphism $\alpha_{2}^{\prime} \in$ $\mathrm{Aut}_{\text {hol }}^{k}\left(\mathbb{C}^{n}\right)$ holomorphically depending on $w \in \mathbb{C}^{k}$ such that $4_{2}, 5_{2}, 6_{2}$ and $7_{2}$ are satisfied with $\epsilon_{2} / 2$ instead of $\epsilon_{2}$, (as we will combine $\alpha^{\prime}$ with another automorphism $\left.\alpha^{\prime \prime}\right)$.

Using Lemma 2.5 we find an automorphism $\alpha_{2}^{\prime \prime} \in \mathrm{Aut}_{h o l}^{k}\left(\mathbb{C}^{n}\right)$ not moving any point of $\alpha_{2}^{\prime}\left(K_{2}\right)$ more than $\epsilon_{2} / 2$, matches the identity up to order $l$ at the points $\left(w, \xi_{i}(w)\right)$ for every $w \in \mathbb{C}^{k}$ and fixing the points $\left(w, a_{j}^{2}\right) j=1, \ldots, k(2)$ for every $w \in \mathbb{C}^{k}$. In addition, for $w \in 2 \overline{\mathbb{B}}_{k}$, the submanifold $\alpha_{2}^{\prime \prime} \circ \alpha_{2}^{\prime} \circ \phi_{1}(w, \tilde{X})$ of $\mathbb{C}^{n}$ do not osculate of order $l$ in any of the points $P_{2}\left(\phi_{1}(w, x)\right)$ with $x \in\left(X_{R_{1}} \cap \tilde{X}\right) \backslash U_{1}$. The composition $\alpha_{2}^{\prime \prime} \circ \alpha_{2}^{\prime}$ will then satisfy $4_{2}, 5_{2}, 6_{2}, 7_{2}$ and $8_{2}$. Finally choose $R_{2}$ so large that $9_{2}$ and $10_{2}$ are satisfied.

The induction for step $s, s>2$, goes exactly as step 2 .

At all steps $s$, we have to make sure that the property not to osculate of order $l$ for $\phi_{s-1} \circ \phi_{0}(\tilde{X})$ is preserved in every point of the image of $\left(X_{R_{s-2}} \cap \tilde{X}\right) \backslash U_{s-2}$. We therefore have to choose $\epsilon_{s} \leq \epsilon_{s-1}$ so small that every perturbation of $\phi_{s-1} \circ \phi_{0}: \tilde{X} \hookrightarrow \mathbb{C}^{n}$ less than $3 \epsilon_{s}$ on the compact $\left(X_{R_{s-2}} \cap \tilde{X}\right) \backslash U_{s-2}$ do not destroy that property.

Because of $5_{\mu}$ and the fact that $\epsilon_{\mu}<\frac{1}{\mu}$ Proposition 4.1 and 4.2 from [14] gives that $A=\lim _{\mu \rightarrow \infty} A_{\mu}(w, z)=\left(w, \lim _{\mu \rightarrow \infty} \tilde{A}_{\mu}(w, z)\right)$ exists uniformly on compacts on $\Omega=\cup_{\mu=1}^{\infty} A_{\mu}^{-1}\left(\mu \mathbb{B}_{k} \times \mu \mathbb{B}_{n}\right)$ and defines a biholomorphic mapping from $\Omega$ onto $\mathbb{C}^{k} \times \mathbb{C}^{n}$. By $4_{\mu}$ the set $\mathbb{C}^{k} \times X$ is contained in $\Omega$. Since $A_{\mu} \in \mathrm{Aut}_{h o l}^{k}\left(\mathbb{C}^{n}\right)$ for each fixed $w_{0} \in \mathbb{C}^{k}$ the map $A_{w_{0}}: \Omega_{w_{0}} \rightarrow \mathbb{C}^{n}$, given by $(w, z) \mapsto \tilde{A}(w, z)$, is a biholomorphic mapping from $\Omega_{w_{0}}=\left\{z \in \mathbb{C}^{n}:\left(w_{0}, z\right) \in \Omega\right\}$ onto $\mathbb{C}^{n}$, and $\Omega_{w_{0}}$ contains $X$. Therefore for all $w_{0} \in \mathbb{C}^{k}$ the map $\phi_{w_{0}}$ defined by $x \mapsto \phi\left(w_{0}, x\right)$ is a proper holomorphic embedding of $X$.

We will now confirm that the constructed embedding satisfies the theorem. Property $3_{\mu}$ and $6_{\mu}$ gives for every $n \in \mathbb{N}$ that $\phi\left(w, x_{j}^{n}\right)=$ $\left(w, a_{j}^{n}\right)$ for every $w \in \mathbb{C}^{k}$ and $j=1,2, \ldots, k(\mu)$.

Define $\epsilon=\sum_{i=2}^{\infty} \epsilon_{i}$, condition $1_{\mu}$ gives that $\epsilon<\frac{1}{2}$.

Now suppose that there is a non-degenerate holomorphic mapping $F: \mathbb{C}^{n} \rightarrow \mathbb{C}^{n}$ and that there are two values $w_{1} \neq w_{2} \in \mathbb{C}^{k}$ of the parameter space such that $F^{-1}\left(\mathbb{C}^{n} \backslash \phi_{w_{2}}(X)\right)=\mathbb{C}^{n} \backslash \phi_{w_{1}}(X)$ and that $\phi_{w_{2}}^{-1} \circ F \circ \phi_{w_{1}}=t$ for some $t \in T$, i.e. some element of the family of automorphisms of $X$. In particular this will hold if for $w_{1} \neq w_{2}$ the embeddings $\phi_{w_{1}}$ and $\phi_{w_{2}}$ are equivalent.

By moving the origin by an arbitrary small translation, we can assume that $J F(0) \neq 0$. Let $\beta=\prod_{i=2}^{\infty}\left(1-\frac{\epsilon_{i}}{2}\right)>0$ and let $\nu_{0}$ be a number 
so large that for every $\nu \geq \nu_{0}$ we have that $t \in T_{\nu}, J F(0)>\frac{1}{(\nu \beta)^{n}}$, $F(0) \in \frac{\nu+2}{2} \mathbb{B}$ and $w_{1}, w_{2} \in \nu \mathbb{B}_{k}$.

For a given $\nu \geq \nu_{0}$ choose a natural number $k$ such that $F\left(\nu \beta \mathbb{B}_{n}\right) \subset$ $(k+2) \mathbb{B}_{n}$ and $k>\nu+2$. Define $F_{j}(z): \mathbb{B}_{n} \rightarrow(k+2) \mathbb{B}_{n}$ by $F_{j}(z)=F(z$. $\left.\nu \prod_{l=j+1}^{k}\left(1-\frac{\epsilon_{l}}{2}\right)\right)$ for $j=1,2, \ldots, k$. For some fix $j \in\{\nu+1, \ldots, k\}$ we have that $F_{j}\left(\mathbb{B}_{n}\right)=F\left(\nu \prod_{l=j+1}^{k}\left(1-\frac{\epsilon_{l}}{2}\right) \mathbb{B}_{n}\right) \subset F\left(\nu \mathbb{B}_{n}\right)$, which by Lemma 3.2 implies that $F_{j}\left(\mathbb{B}_{n}\right)$ do not contain any point $a_{l}^{j} \in \partial(j+1) \mathbb{B}_{n}$, $l=1,2, \ldots, k(j)$ and $j \geq \nu+1$.

In addition we have

$$
\left|J F_{j}(0)\right|=\left|\nu^{n} \prod_{l=j+1}^{k}\left(1-\frac{\epsilon_{l}}{2}\right)^{n} J F(0)\right|>\nu^{n} \beta^{n} J F(0)>1
$$

and $F_{j}(0)=F(0) \in \frac{j+1}{2} \mathbb{B}_{n}$. Property $2_{k}$ now gives that $F_{k}((1-$ $\left.\left.\frac{\epsilon_{k}}{2}\right) \mathbb{B}_{n}\right)=F_{k-1}\left(\mathbb{B}_{n}\right) \subset(k+1) \mathbb{B}_{n}$. Induction from $k$ down to $\nu+1$ gives that $F_{j-1}\left(\mathbb{B}_{n}\right) \subset(j+1) \mathbb{B}_{n}$ so, for $j=\nu+1$ we have $F_{\nu}\left(\mathbb{B}_{n}\right) \subset(\nu+2) \mathbb{B}_{n}$ and therefore $F\left(\beta \nu \mathbb{B}_{n}\right) \subset(\nu+2) \mathbb{B}_{n}$ for every $\nu \geq \nu_{0}$. This growth condition implies that $F$ is an affine mapping, and the fact that $F$ is non-degenerate means that $F$ is an affine automorphism.

Remember that $\left(\varphi_{1}\right)_{w}(\tilde{X})$ osculates of order $l$ at $\xi_{i}(w)$, for $w \in \mathbb{C}^{k}$, and this is preserved by $7_{\mu}$ over the induction. Hence, we see that $\varphi_{w}(\tilde{X})$ osculates of order $l$ at $\varphi\left(x_{i}\right)=\xi_{i}(w), i=1,2, \ldots, m$. From property $8_{\mu}$ it follows that $\varphi_{w}(\tilde{X})$ do not osculate of order $l$ in any other point. Due to this, since the affine automorphism $F$ maps $\varphi_{w_{1}}(\tilde{X})$ to $\varphi_{w_{2}}(\tilde{X})$, it also maps the set $\left\{\xi_{i}\left(w_{1}\right)\right\}_{i=1}^{m}$ to the set $\left\{\xi_{i}\left(w_{2}\right)\right\}_{i=1}^{m}$. By the choice of $\left\{\xi_{i}(w)\right\}$ there is no such automorphism, see Proposition 2.1 .

Lemma 3.1. In the notation of the proof of Theorem 1.1 holds:

$$
\begin{array}{r}
\varphi^{-1}\left((\nu-1) \overline{\mathbb{B}}_{k} \times(\nu-1) \mathbb{B}_{n}\right) \subset P_{2}\left(\left(A_{\nu} \circ \phi_{0}\right)^{-1}\left((\nu-1) \overline{\mathbb{B}}_{k} \times \nu \mathbb{B}_{n}\right)\right)= \\
=P_{2}\left(\phi_{\nu}^{-1}\left((\nu-1) \overline{\mathbb{B}}_{k} \times \nu \mathbb{B}_{n}\right)\right)
\end{array}
$$

Proof. Let $w \in(\nu-1) \overline{\mathbb{B}}_{k}$ be a fix point and consider some $x \in \varphi_{w}^{-1}((\nu-$ $\left.1) \overline{\mathbb{B}}_{n}\right)$, where $\varphi_{w}^{-1}$ is the restriction of $\varphi^{-1}$ to $\{w\} \times \mathbb{C}^{n}$. This implies that $\varphi_{w}(x) \in(\nu-1) \overline{\mathbb{B}}_{n}$. Now choose $k_{0}>\nu$ and $0<\delta<1-2 \epsilon$ such that

$$
\phi_{k, w}(x) \in(\nu-1+\delta) \overline{\mathbb{B}}_{n} \text { for every } k \geq k_{0} .
$$

Property $5_{\nu+1}$ gives that $\left\|\alpha_{\nu+1}(w, z)-(w, z)\right\| \leq \epsilon_{\nu+1}$ for $(w, z) \in$ $\left.(\nu+1) \overline{\mathbb{B}}_{k} \times \overline{\mathbb{B}}_{n}\right)$ and Rouchés theorem that $\alpha_{\nu+1, w}\left(\nu \mathbb{B}_{n}\right) \supset\left(\nu-2 \epsilon_{\nu}\right) \mathbb{B}_{n}$, 
see Remark 6, or in other words $\nu \mathbb{B}_{n} \supset\left(\alpha_{\nu+1, w}\right)^{-1}\left(\left(\nu-2 \epsilon_{\nu}\right) \mathbb{B}_{n}\right)$ so

$$
\begin{array}{r}
\left(\phi_{\nu}\right)_{w}^{-1}\left(\nu \mathbb{B}_{n}\right)=\left(A_{\nu} \circ \phi_{0}\right)_{w}^{-1}\left(\nu \mathbb{B}_{n}\right) \supset\left(A_{\nu+1} \circ \phi_{0}\right)_{w}^{-1}\left(\left(\nu-2 \epsilon_{\nu}\right) \mathbb{B}_{n}\right)= \\
=\left(\phi_{\nu+1}\right)_{w}^{-1}\left(\left(\nu-2 \epsilon_{\nu}\right) \mathbb{B}_{n}\right) .
\end{array}
$$

Induction using $5_{\nu+2}, \ldots, 5_{k}$ gives

$$
\begin{aligned}
\left(A_{\nu} \circ \phi_{0}\right)_{w}^{-1}\left(\nu \mathbb{B}_{n}\right) \supset\left(A_{k} \circ \phi_{0}\right)_{w}^{-1}\left(\left(\nu-2 \sum_{l=\nu}^{k-1} \epsilon_{l}\right) \mathbb{B}_{n}\right) \supset & \\
& \supset\left(A_{k} \circ \phi_{0}\right)_{w}^{-1}\left((\nu-2 \epsilon) \mathbb{B}_{n}\right) .
\end{aligned}
$$

By our choice of $\delta$ we have $\nu-2 \epsilon>\nu-1+\delta$, so (3) and (41) implies that $\phi_{\mu}^{w}(x) \in \nu \mathbb{B}_{n}$.

Remark 6 . To see that $\alpha_{\nu+1, w}\left(\nu \mathbb{B}_{n}\right) \supset\left(\nu-2 \epsilon_{\nu}\right) \mathbb{B}_{n}$ holds, we consider the following situation, $\left\|\alpha_{q}(w, z)-(w, z)\right\| \leq \epsilon_{q}$ for $(w, z) \in q \overline{\mathbb{B}}_{k} \times q \overline{\mathbb{B}}_{n}$.

$$
\begin{aligned}
& \left\|\alpha_{q}(w, z)-(w, z)\right\|=\left\|\alpha_{q}(w, z)-(w, p)-((w, z)-(w, p))\right\| \leq \\
& \epsilon_{q}<\|(w, z)-(w, p)\| \text { for } z \in q \overline{\mathbb{B}}_{k} \times \partial q \overline{\mathbb{B}}_{n} \text { and } p \in\left(q-2 \epsilon_{q}\right) \overline{\mathbb{B}}_{n} .
\end{aligned}
$$

Since $(w, z)-(w, p)$ has a root and consequently, by Rouché, $\alpha_{q}(w, z)-$ $(w, p)$ will too. So for every $p \in\left(q-2 \epsilon_{q}\right) \overline{\mathbb{B}}_{n}$ we always have a solution to the equation $\alpha_{q}(w, z)=(w, p)$ for some $z \in q \overline{\mathbb{B}}_{n}$, therefore we draw the conclusion that $\alpha_{\nu+1, w}\left(\nu \mathbb{B}_{n}\right) \supset\left(\nu-2 \epsilon_{\nu}\right) \mathbb{B}_{n}$.

Lemma 3.2. In the notation of the proof of Theorem 1.1 holds: For every $j \geq \nu+1$ we have that $F\left(\nu \mathbb{B}_{n}\right) \cap \cup_{l=1}^{k(j)}\left\{a_{l}^{j}\right\}=\emptyset$.

Proof. Suppose, to reach a contradiction, that there exist $z \in \nu \mathbb{B}_{n}$ such that $F(z)=a_{l}^{j}$ for some $j \geq \nu+1$ and some $l$ between 1 and $k(j)$. Since $F^{-1}\left(\mathbb{C}^{n} \backslash \varphi_{w_{2}}(X)\right)=\mathbb{C}^{n} \backslash \varphi_{w_{1}}(X)$, we have that $z \in \varphi_{w_{1}}(X)$.

Let $x=\varphi_{w_{1}}^{-1}(z) \in \varphi_{w_{1}}^{-1}\left(\nu \mathbb{B}_{n}\right)$, which gives $F \circ \varphi_{w_{1}}(x)=a_{l}^{j}=\varphi_{w_{2}}\left(x_{l}^{j}\left(w_{2}\right)\right)$. Thus $t(x)=\varphi_{w_{2}}^{-1} \circ F \circ \varphi_{w_{1}}(x)=x_{l}^{j}\left(w_{2}\right)$. Using Lemma 3.1 we conclude that $x \in P_{2}\left(\phi_{\nu+1, w_{1}}^{-1}\left((\nu+1) \overline{\mathbb{B}}_{n}\right)\right)$ and with $t \in T_{\nu}$ and $w_{1} \in \nu \mathbb{B}_{k}$ it follows that

$$
\rho(t(x)) \leq \max _{\phi_{\nu+1}^{-1}(\nu+1) \overline{\mathbb{B}}_{n}, t \in T_{\nu}, w \in \nu \mathbb{B}_{k}} \rho(t(y)) .
$$

Since $j \geq \nu+1$ we have

$$
\rho(t(x)) \leq \max _{\phi_{j, w}^{-1} j \overline{\mathbb{B}}_{n}, t \in T_{j-1}, w \in(j-1) \mathbb{B}_{k}} \rho(t(y)),
$$

(for $j>\nu+1$ we have that $\phi_{j}$ maps the inverse image $\phi_{j-1}^{-1}\left((j-1) \mathbb{B}_{n}\right)$ into $\left.\left(1+\epsilon_{j}\right)(j-1) \mathbb{B}_{n} \subset j \mathbb{B}_{n}\right)$. However condition $3_{j}$ gives

$$
\rho\left(x_{l}^{j}(w)\right)>\max _{y \in \phi_{j-1, w}^{-1}\left(j \overline{\mathbb{B}}_{k} \times j \overline{\mathbb{B}}_{n}\right), t \in T_{j}} \rho(t(y)) .
$$


Therefore $\rho\left(x_{l}^{j}\left(w_{2}\right)\right)>\rho(t(x))$, which contradicts $t(x)=x_{l}^{j}\left(w_{2}\right)$.

\section{EISENMAN HYPERBOLICITY OF THE EMBEDDINGS}

Let $M$ be a complex manifold of dimension $n$. We denote the holomorphic tangent bundle of $M$ by $T M$ and the holomorphic tangent space at $p \in M$ by $T_{p} M$. The $k$-th exterior power of $T_{p} M$ and $T M$ will be denoted by $\bigwedge^{k} T_{p} M$ and $\bigwedge^{k} T M$. Let also $D_{p}^{k} M$ and $D^{k} M$ denote the set of decomposable elements in $\bigwedge^{k} T_{p} M$ and $\bigwedge^{k} T M$.

Recall that the Eisenman $n$-norm for a $u \in D_{p}^{n} M$ is defined as [10], 22

$E_{n}^{M}(p, u)=\inf \left\{\|v\|^{2}: v \in D_{0}^{n} \mathbb{B}_{n}, \exists F \in \mathcal{O}\left(\mathbb{B}_{n}, M\right), F(0)=p, F_{*} v=u\right\}$.

A complex manifold is called $n$-Eisenman hyperbolic if $E_{n}^{M}(p, u)>0$ for all $p \in M$ and all non-zero $u \in D_{p}^{n} M$. Compare with [5].

We use the notation from the proof of Theorem 1.1.

Theorem 4.1 (Addition to Theorem 1.1). For all $w \in \mathbb{C}^{k}$ the complement $\mathbb{C}^{n} \backslash \varphi_{w}(X)$ of the embedding $\varphi_{w}(X)$ is Eisenman n-hyperbolic.

Proof. Suppose there exists a point $p \in \mathbb{C}^{n} \backslash \varphi_{w}(X)=M$ such that $E_{n}^{M}(p, u)=0$ for the (unique up to a constant) non-zero $u \in D_{p}^{n} M$. This means that

$$
\inf _{f} \frac{1}{|J f(0)|^{2}}\left(\frac{i}{2}\right)^{n} d z \wedge d \bar{z}=0
$$

for some point $p$ where $f \in \mathcal{O}\left(\mathbb{B}_{n}, M\right)$ such that $f(0)=p$ and $f_{*}\left(T_{0} \mathbb{B}_{n}\right)=$ $v$.

Let $\nu \in \mathbb{N}$ be a fixed number such that $p \in \frac{\nu+2}{2} \mathbb{B}_{n}$. By (5) there is $F: \mathbb{B}_{n} \rightarrow M$ such that $F(0)=p$ and $J F(0)$ is arbitrary large, for example

$$
J F(0)>\max \left(\frac{1}{\beta^{n}},(\nu+2)^{n} \beta^{n}\right)
$$

There is an $\alpha \in \mathbb{C}, 0<\alpha<1$ so that $J F(0)>\frac{1}{\alpha^{n} \beta^{n}}$. Since $F\left(\alpha \overline{\mathbb{B}}_{n}\right)$ is compact we find $k \in \mathbb{N}$ such that $F\left(\alpha \mathbb{B}_{n}\right) \subset(k+2) \mathbb{B}_{n}$.

Define $F_{j}(z)=F\left(\alpha \prod_{l=j+1}^{k}\left(1-\frac{\epsilon_{j}}{2}\right) z\right)$. It holds:

$J F_{j}(0) \geq \alpha^{n} \beta^{n} J F(0)>1$ for every $j$, also for $\nu+1 \leq j \leq k$ we have $F_{j}(0)=p \in \frac{\nu+2}{2} \mathbb{B}_{n}$ and $F_{j}\left(\mathbb{B}_{n}\right)$ obviously does not meet the points $a_{l}^{j}, l=1,2, \ldots, k(j)$, (for $j$ large enough as in the proof of the main theorem).

We conclude inductively by property $2_{j} F_{j-1}\left(\mathbb{B}_{n}\right) \subset(j+1) \mathbb{B}_{n}$ for $\nu+1 \leq j \leq k$. This means in particular $F_{\nu}\left(\mathbb{B}_{n}\right) \subset(\nu+2) \mathbb{B}_{n}$ which 
implies

$$
J F_{\nu}(0) \leq(\nu+2)^{n}
$$

and therefore

$$
J F(0) \leq(\nu+2)^{n} \alpha^{n} \beta^{n} .
$$

This contradicts (6), thus $\mathbb{C}^{n} \backslash \varphi_{w}(X)$ is Eisenman $n$-hyperbolic.

That Eisenman hyperbolic manifold have a cancellation property was used in [4], Theorem 1.10., (for a simple proof see for example [4])

Proposition 4.1. Let $Y$ and $Z$ be $n$-Eisenman hyperbolic manifolds. Then any biholomorphic map $\Psi=\left(\psi_{1}, \psi_{2}\right): Y \times \mathbb{C}^{l} \rightarrow Z \times \mathbb{C}^{l}$ is of the form $\Psi(y, z)=\left(\psi_{1}(y), \psi_{2}(y, z)\right)$, where $\psi_{1}: Y \rightarrow Z$ is biholomorphic.

With Proposition 4.1 and Theorem 4.1 we get

Theorem 4.2. Let $X$ be a complex space, which can be embedded in $\mathbb{C}^{n}$ and such that the group of holomorphic automorphisms $\operatorname{Aut}_{\text {hol }}(X)$ is a Lie group. Then there exist, for $k=n-1-\operatorname{dim} X$, a family of holomorphic embeddings of $X \times \mathbb{C}^{l}$ into $\mathbb{C}^{n} \times \mathbb{C}^{l}$ parameterized by $\mathbb{C}^{k}$, such that for different parameters $w_{1} \neq w_{2} \in \mathbb{C}^{k}$ the embeddings $\psi_{w_{1}}, \psi_{w_{2}}: X \times \mathbb{C}^{l} \hookrightarrow \mathbb{C}^{n+l}$ are non-equivalent (up to automorphisms).

Proof. Take $\varphi$ from Theorem 1.1 and consider

$$
\Psi: \mathbb{C}^{k} \times X \times \mathbb{C}^{l} \rightarrow \mathbb{C}^{k} \times \mathbb{C}^{n} \times \mathbb{C}^{l}
$$

defined by

$$
(w, x, y) \mapsto(w, \varphi(w, x), y)=(w, \psi(w, x, y)) .
$$

Assume that the embeddings $\psi_{w_{1}}$ and $\psi_{w_{2}}$, where $w_{1} \neq w_{2}$ are equivalent. This means that there exists an automorphism $\alpha \in \operatorname{Aut}_{\text {hol }}\left(\mathbb{C}^{n+l}\right)$ such that $\alpha\left(\varphi_{w_{1}}(X) \times \mathbb{C}^{l}\right)=\varphi_{w_{2}}(X) \times \mathbb{C}^{l}$ and therefore the same for the complements $\alpha\left(\left(\mathbb{C}^{n} \backslash \varphi_{w_{1}}(X)\right) \times \mathbb{C}^{l}\right)=\left(\mathbb{C}^{n} \backslash \varphi_{w_{2}}(X)\right) \times \mathbb{C}^{l}$.

Now by Proposition 4.1 there exists $\alpha_{1} \in \operatorname{Aut}_{\text {hol }}\left(\mathbb{C}^{n}\right)$ such that $\alpha_{1}\left(\mathbb{C}^{n} \backslash \varphi_{w_{1}}(X)\right)=\mathbb{C}^{n} \backslash \varphi_{w_{2}}(X)$. Thus $\alpha_{1}\left(\varphi_{w_{1}}(X)\right)=\varphi_{w_{2}}(X)$, which contradicts the choice of $\varphi$.

A special case which is worth to state separately is $X=\mathbb{C}$.

Corollary 4.1. There exist, for $k=n-l-1$, a family of holomorphic embeddings of $\mathbb{C}^{l}$ into $\mathbb{C}^{n}$ parameterized by $\mathbb{C}^{k}$, such that for different parameters $w_{1} \neq w_{2} \in \mathbb{C}^{k}$ the embeddings $\psi_{w_{1}}, \psi_{w_{2}}: \mathbb{C}^{l} \hookrightarrow \mathbb{C}^{n}$ are non-equivalent.

We end this section with a little trick showing that one can even have families of pairwise non-equivalent embeddings containing the standard 
embedding as a member of the family. Note that the embeddings constructed in the proof of Theorem 1.1 are not containing the standard embedding since the complement of each embedding is $n$-Eisenman hyperbolic.

Proposition 4.2. For each $0<l<n-1$ there is a holomorphic family of holomorphic embeddings of $\mathbb{C}^{l}$ into $\mathbb{C}^{n}$ parameterized by $\mathbb{C}$, such that for different parameters $w_{1} \neq w_{2} \in \mathbb{C}$ the embeddings $\psi_{w_{1}}, \psi_{w_{2}}: \mathbb{C}^{l} \hookrightarrow$ $\mathbb{C}^{n}$ are non-equivalent. Moreover for the embedding $\psi_{0}$ is equivalent to the standard embedding.

Proof. Take a family $\Phi: \mathbb{C} \times \mathbb{C} \rightarrow \mathbb{C} \times \mathbb{C}^{n-l+1} \Psi_{0}(w, z)=(w, \phi(w, z))$ as constructed in Theorem 1.1 and cross it with $\mathbb{C}^{l-1}$ as in Theorem 4.2 to get a family $\Psi_{1}: \mathbb{C} \times \mathbb{C}^{l} \rightarrow \mathbb{C} \times \mathbb{C}^{n} \Psi_{1}(w, x)=\left(w, \phi_{1}(w, x)\right)$. By using a translation we can assume that $\phi_{1}(w, 0)=0 \forall w \in \mathbb{C}$.

Now define the family $\Psi: \mathbb{C} \times \mathbb{C}^{l} \rightarrow \mathbb{C} \times \mathbb{C}^{n}$ by

$$
\Psi(w, x)=\left(w, \frac{1}{w} \phi_{1}(w, w x)\right)=:(w, \psi(w, x))
$$

for $w \neq 0$ and by its obvious limit $x \mapsto \phi_{1}^{\prime}(0,0) x$ for $w=0$. Thus for $w=0$ we have the standard embedding in the family. All other members $\psi_{w}$ of the family are by definition equivalent to the embedding $\phi_{w}$ and therefore pairwise non-equivalent. No member in the family except $\psi_{0}$ is equivalent to the standard embedding since otherwise there would exist a holomorphic map of rank $n-l+1$ into the complement of $\phi_{w}(\mathbb{C})$ which contradicts the Eisenman $n-l+1$-hyperbolicity (Theorem 4.11).

\section{FAmilies of holOmorphic $\mathbb{C}^{*}$-ACTIONS ON AFFine SPACE}

In this section we employ the method from [8] and [9] to construct (non-linearizable) $\mathbb{C}^{*}$-actions on affine spaces out of embeddings $\mathbb{C}^{l} \hookrightarrow$ $\mathbb{C}^{n}$. We will not give all proofs in detail. The important point we want to check here is that if the embeddings are holomorphically parametrized, then the resulting $\mathbb{C}^{*}$-actions depend holomorphically on the parameter.

Let's go through the method:

For an embedding $\varphi: \mathbb{C}^{l} \rightarrow \mathbb{C}^{n}$ take generators of the ideal $I_{\varphi\left(\mathbb{C}^{l}\right)}<$ $\mathcal{O}\left(\mathbb{C}^{n}\right)$ of the image manifold, say $f_{1}, \ldots, f_{N} \in \mathcal{O}\left(\mathbb{C}^{n}\right)$ (in this case $N=n-l$ would be sufficient, since $\mathbb{C}^{l}$ is always a complete intersection in $\mathbb{C}^{n}$ by results of Forster and Ramspott [13], but this is not important for the construction) and consider the manifold 


$$
\begin{aligned}
M:=\left\{\left(z_{1}, \ldots, z_{n}, u_{1}, \ldots u_{N}, v\right)\right. & \in \mathbb{C}^{n+N+1}: \\
f_{i}\left(z_{1}, \ldots, z_{n}\right) & \left.=u_{i} v \quad \forall i=1, \ldots, N\right\}
\end{aligned}
$$

which in [8] is called Rees space. This notion was introduced there by the authors since they were not aware of the fact that this is a wellknown construction, called affine modification, going back to Oscar Zariski. Geometrically the manifold $M$ results from $\mathbb{C}_{z, v}^{n+1}$ by blowing up along the center $\mathcal{C}=\varphi\left(\mathbb{C}^{l}\right) \times 0_{v}$ and deleting the proper transform of the divisor $\mathcal{D}=\{v=0\}$. Since our center is not algebraic but analytic, the process usually is called pseudo-affine modification.

Lets denote the constructed manifold $M$ by $\operatorname{Mod}\left(\mathbb{C}^{n+1}, \mathcal{D}, \mathcal{C}\right)=$ $\operatorname{Mod}\left(\mathbb{C}_{z, v}^{n+1},\{v=0\}, \varphi\left(\mathbb{C}^{l}\right) \times\{v=0\}\right)$. It's clear from the geometric description that the resulting manifold does not depend on the choice of generators for the ideal $I_{\mathcal{C}}$ of the center.

The important fact about the above modifications is that $\operatorname{Mod}\left(\mathbb{C}_{z, v}^{n+1},\{v=0\}, \varphi\left(\mathbb{C}^{l}\right) \times\{v=0\}\right) \times \mathbb{C}^{l}$ is biholomorphic to $\mathbb{C}^{n+l+1}$ $\cong \operatorname{Mod}\left(\mathbb{C}_{z, u, v}^{n+l+1},\{v=0\}, \varphi\left(\mathbb{C}^{l}\right) \times 0_{u} \times 0_{v}\right)$. The later biholomorphism comes from the fact that there is an automorphism of $\mathbb{C}^{n+l+1}$ leaving the divisor $\{v=0\}$ invariant and straightening the center $\varphi\left(\mathbb{C}^{l}\right) \times 0_{v}$ inside the divisor (see Lemma 2.5. in [8]).

Lets check that this important fact depends holomorphically on the parameter.

Lemma 5.1. Let $\Phi_{1}: \mathbb{C}^{k} \times X \hookrightarrow \mathbb{C}^{k} \times \mathbb{C}^{n}, \Phi_{1}(w, x)=\left(w, \varphi_{1}(w, x)\right)$ and $\Phi_{2}: \mathbb{C}^{k} \times X \hookrightarrow \mathbb{C}^{k} \times \mathbb{C}^{m}, \Phi_{2}(w, x)=\left(w, \varphi_{2}(w, x)\right)$ be two holomorphic families of proper holomorphic embeddings of a complex space $X$ into $\mathbb{C}^{n}$ resp. $\mathbb{C}^{m}$ parametrized by $\mathbb{C}^{k}$. Then there is an automorphism $\alpha$ of $\mathbb{C}^{n+m}$ parametrized by $\mathbb{C}^{k}$, i.e. $\alpha \in$ Aut $_{\text {hol }}\left(\mathbb{C}_{w}^{k} \times \mathbb{C}_{z}^{n+m}\right)$ with $\alpha(w, z)=(w, \tilde{\alpha}(w, z))$, such that $\alpha \circ\left(\Phi_{1} \times 0_{m}\right)=0_{n} \times \Phi_{2}$.

Proof. By an application of Theorem B the holomorphic map $\varphi_{1}$ : $\mathbb{C}^{k} \times X$ to $\mathbb{C}^{n}$ extends to a holomorphic map $\mu_{1}$ from $\mathbb{C}^{k} \times \mathbb{C}^{m} \supset$ $\Phi_{2}\left(\mathbb{C}^{k} \times X\right)$ to $\mathbb{C}^{n}$ (so $\left.\mu_{1} \circ \varphi_{2}=\varphi_{1}\right)$. Likewise there is a holomorphic map $\mu_{2}: \mathbb{C}^{k} \times \mathbb{C}^{n} \rightarrow \mathbb{C}^{m}$ with $\mu_{2} \circ \varphi_{1}=\varphi_{2}$. Define the parametrized automorphisms $\alpha_{1}, \alpha_{2}$ of $\mathbb{C}^{k} \times \mathbb{C}^{n} \times \mathbb{C}^{m}$ by $\alpha_{1}(w, z, y)=(w, z, y+$ $\left.\mu_{2}(w, z)\right)$ and $\alpha_{2}(w, z, y)=\left(w, z+\mu_{1}(w, y), y\right)$. Now $\alpha=\alpha_{2}^{-1} \circ \alpha_{1}$ is the desired automorphism.

Lemma 5.2. Let $\Phi: \mathbb{C}^{k} \times \mathbb{C}^{l} \hookrightarrow \mathbb{C}^{k} \times \mathbb{C}^{n} \Phi(w, \theta)=(w, \varphi(w, \theta))$ be a holomorphic family of proper holomorphic embeddings of $\mathbb{C}^{l}$ into $\mathbb{C}^{n}$ parametrized by $\mathbb{C}^{k}$. 
Then $\operatorname{Mod}\left(\mathbb{C}_{w, z, v}^{k+n+1},\{v=0\}, \Phi\left(\mathbb{C}^{k} \times \mathbb{C}^{l}\right) \times\{v=0\}\right) \times \mathbb{C}^{l} \cong \mathbb{C}^{k+n+l+1}$. Moreover there is a biholomorphism such that the restriction to each fixed parameter $w \in \mathbb{C}^{k}$ is a biholomorphism from $\operatorname{Mod}\left(\mathbb{C}_{z, v}^{n+1},\{v=\right.$ $\left.0\}, \Phi\left(\{w\} \times \mathbb{C}^{l}\right) \times\{v=0\}\right) \times \mathbb{C}^{l} \cong \mathbb{C}^{n+l+1}$.

Proof. Apply Lemma 5.1 to the families $\Phi_{1}=\Phi$ and $\Phi_{2}$ the trivial family $\Phi_{2}: \mathbb{C}^{k} \times \mathbb{C}^{l} \hookrightarrow \mathbb{C}^{k} \times \mathbb{C}^{l} \Phi_{2}(w, \theta)=(w, \theta)$. Let $\alpha \in$ Aut $_{\text {hol }}\left(\mathbb{C}^{k} \times \mathbb{C}^{n} \times \mathbb{C}^{l}\right)$ be the resulting parametrized automorphism which we extend to $\mathbb{C}^{k+n+l+1}$ by letting it act trivial on the last coordinate $v$. Then by definition $\operatorname{Mod}\left(\mathbb{C}_{w, z, v}^{k+n+1},\{v=0\}, \Phi\left(\mathbb{C}^{k} \times \mathbb{C}^{l}\right) \times\{v=\right.$ $0\}) \times \mathbb{C}^{l}=\operatorname{Mod}\left(\mathbb{C}_{w, z, \theta, v}^{k+n+l+1},\{v=0\}, \Phi\left(\mathbb{C}^{k} \times \mathbb{C}^{l}\right) \times\{v=0\} \times 0_{l}\right)$ and applying (the extended) $\alpha$ we get that the later is biholomorphic to $\operatorname{Mod}\left(\mathbb{C}_{w, z, \theta, v}^{k+n+l+1},\{v=0\}, \mathbb{C}_{w}^{k} \times 0_{n} \times \mathbb{C}_{\theta}^{l} \times\{v=0\}\right)$. The last manifold is obviously biholomorphic to $\mathbb{C}^{k+n+l+1}$ since blowing up along a straight center and deleting the proper transform of a straight divisor does not change the affine space. The above constructed biholomorphism restricts to each fixed parameter as desired since $\alpha$ is a parametrized automorphism. This can be also seen by writing down concrete formulas for the modifications using generators $f_{1}(w, z), \ldots, f_{N}(w, z)$ of the ideal $I_{\phi\left(\mathbb{C}^{k} \times \mathbb{C}^{l}\right)}$ in $\mathcal{O}\left(\mathbb{C}^{k+n}\right)$ and remarking that for each fixed $w \in \mathbb{C}^{k}$ the functions $f_{1}(w, \cdot), \ldots, f_{N}(w, \cdot)$ generate the ideal $I_{\Phi_{w}\left(\mathbb{C}^{l}\right)}$.

Now we describe the group actions:

Let $f_{1}(w, z), \ldots, f_{N}(w, z)$ be generators of the ideal $I_{\phi\left(\mathbb{C}^{k} \times \mathbb{C}^{l}\right)}$ in $\mathcal{O}\left(\mathbb{C}^{k+n}\right)$ and consider $\operatorname{Mod}\left(\mathbb{C}_{w, z, v}^{k+n+1},\{v=0\}, \Phi\left(\mathbb{C}^{k} \times \mathbb{C}^{l}\right) \times\{v=0\}\right) \times \mathbb{C}^{l} \cong$ $\mathbb{C}^{k+n+l+1}$ as the affine manifold given by equations:

$$
\left\{(w, z, v, u) \in \mathbb{C}^{k} \times \mathbb{C}^{n} \times \mathbb{C} \times \mathbb{C}^{N}: f_{i}(w, z)=u_{i} v \quad \forall i=1, \ldots, N\right\} \times \mathbb{C}_{x}^{l}
$$

On it we consider the action of $\mathbb{C}_{\nu}^{*}$ given by the restriction of the following linear action on the ambient space:

$$
\begin{aligned}
& \mathbb{C}^{*} \times \mathbb{C}^{k} \times \mathbb{C}^{n} \times \mathbb{C} \times \mathbb{C}^{N} \times \mathbb{C}^{l} \rightarrow \mathbb{C}^{k} \times \mathbb{C}^{n} \times \mathbb{C} \times \mathbb{C}^{N} \times \mathbb{C}^{l} \\
&(\nu,(w, z, v, u, x)) \mapsto\left(w, z, \nu^{2} v, \nu^{-2} u_{1}, \ldots, \nu^{-2} u_{N}, \nu x_{1}, \ldots, \nu x_{l}\right)
\end{aligned}
$$

This gives by Lemma 5.2 a holomorphic family of $\mathbb{C}^{*}$-actions on $\mathbb{C}^{n+l+1}$ parametrized by $\mathbb{C}^{k}$, i.e., an action $\mathbb{C}^{*} \times \mathbb{C}^{k} \times \mathbb{C}^{n+l+1} \rightarrow \mathbb{C}^{k} \times$ $\mathbb{C}^{n+l+1}$ of the form $(\nu(w, z)) \mapsto(w, \nu(w, z))$. Calculating (as in [9]) the Luna-stratification of the categorical quotient $\mathbb{C}^{n+l+1} / / \mathbb{C}^{*}$ for the $\mathbb{C}^{*}$ action for fixed $w$, in particular the inclusion of the fixed point stratum in the $\mathbb{Z} / 2 \mathbb{Z}$-isotropy stratum one sees that this inclusion is biholomorphic to $\Phi_{w}\left(\mathbb{C}^{l}\right) \subset \mathbb{C}^{n}$. Thus if for different parameters $w_{1} \neq w_{2}$ there 
were an equivariant automorphism $\alpha \in \operatorname{Aut}_{h o l}\left(\mathbb{C}^{n}\right)$ the induced isomorphism of the categorical quotients would map the Luna-stratifications onto each other. Therefore the restriction of that induced isomorphism to the $\mathbb{Z} / 2 \mathbb{Z}$-isotropy stratum would give an automorphism $\beta$ of $\mathbb{C}^{n}$ with $\beta\left(\Phi_{w_{1}}\left(\mathbb{C}^{l}\right)\right)=\Phi_{w_{2}}\left(\mathbb{C}^{l}\right)$. This shows that pairwise non-equivalent embeddings lead to non-equivalent $\mathbb{C}^{*}$-actions. Combining this with Theorem 1.1 (embeddings of $\mathbb{C}$ into $\mathbb{C}^{n}$ parametrized by $\mathbb{C}^{n-2}$ for $n \geq 3$ ) we have proved Theorem 1.3 from the introduction. In the same way Theorem 1.4 from the introduction follows from Proposition 4.2. It's an easy exercise that a straight embedding leads to a linear action.

\section{Concluding REMARKS}

Carefully examining the proof of Theorem 1.1 and the proofs of the technical results from section 2 one sees that there is no place where we use the fact that the parameter space is affine space $\mathbb{C}^{k}$. What we use of the parameter space is a graduation (in the proof of the parametrized Andersén-Lempert-theorem) so say an affine algebraic variety would do the job. Most important is the dimension condition $\operatorname{dim} X+\operatorname{dim}$ (parameterspace) $<\mathrm{n}$ (here dimension is always dimension of the smooth part). So in fact we construct families parametrized by any space of the right dimension. The authors wonder whether there is any nice structure on the set of all equivalence classes of proper holomorphic embeddings say of $\mathbb{C}^{l}$ into $\mathbb{C}^{n}$ and how "big" is this set?

Our construction of embeddings used two techniques, the growth restrictions which worked well for embedding manifolds with a "small" automorphism group, namely a Lie group, and the Eisenman hyperbolicity for crossing the situation with affine space. Combining this we got families of embeddings of affine spaces.

What about the number of equivalence classes of proper holomorphic embeddings of other manifolds with infinite-dimensional automorphism groups, e.g. manifolds with the density property, into affine spaces? A concrete question in this direction would be:

How many embeddings of a Danielevski surface $f(X)=$ uv into affine spaces do there exist?

It's known that there exist at least two algebraic embeddings of the Danielevski surface $p(y)=u^{n} v$ (degree of $p$ is at least 2) into $\mathbb{C}^{3}$ which are algebraically non-equivalent, i.e. there is no algebraic automorphism of $\mathbb{C}^{3}$ mapping one image onto the other [20]. In the same paper Freudenburg and Moser show that the constructed embeddings are holomorphically isomorphic using the linearization results of Heinzner and the first author [27]. On the other hand there is a non-standard 
holomorphic embedding of the Danielevski surface into $\mathbb{C}^{3}$, which follows from the ideas of Rosay and Rudin [38]:

Proposition 6.1. Any algebraic subvariety $A$ in $\mathbb{C}^{n}(n \geq 2)$ admits another holomorphic embedding into $\mathbb{C}^{n}$ not isomorphic to the inclusion.

Proof. The restriction of a generic projection onto a hyperplane to $A$ is a proper map. Thus by the results in [38] any discrete sequence of points in $A$ is tame (in fact very tame). Now there is a holomorphic embedding $\varphi$ of $A$ into $\mathbb{C}^{n}$ (constructed by applying a sequence of automorphisms to the inclusion) such that $\varphi(A)$ contains a non tame set $F$ (details as in [16]). The existence of a holomorphic automorphism mapping $A$ onto $\varphi(A)$ contradicts the non tameness of $F$.

It would be interesting to know under which conditions parametrized points (by any parameter space and in any category, continuous, holomorphic differentiable, algebraic) are simultanuously standardizable.

\section{APPENDIX: PROOFS OF TECHNICAL PREPARATIONS}

In this section we give the proofs of the lemmas etc. used in the proof of the main theorem.

\subsection{A parametrized version of the Andersén-Lempert theo-}

rem. Our main technique we use to construct families of embeddings are (compositions of) automorphisms of $\mathbb{C}^{n}$.

The ground-breaking papers of Andersén and Lempert ([1], [2]) established remarkable properties of the automorphism group of $\mathbb{C}^{n}(n \geq$ 2) which imply, in particular, that any local holomorphic phase flow on a Runge domain $\Omega$ in $\mathbb{C}^{n}$ can be approximated by global holomorphic automorphisms of $\mathbb{C}^{n}$ (for an exact statement see Theorem 2.1 in [19]). We will give here a parametrized version of the so called Andersén-Lempert-theorem and in addition we consider the following two geometric structures: that of vector fields vanishing on the first $N$ standard points in $\mathbb{C}^{n}$, and that of vector fields vanishing on the first coordinate axis. Since the parametric version is an easy consequence of the non-parametric version and the fixing of the first $N$-standard points is a special case of Theorem 6 in [32] we just give a small indication of the proof.

Theorem 7.1 (Andersén-Lempert-theorem with parameter and fixing finitely many points). Let $\Omega$ be an open set in $\mathbb{C}^{k} \times \mathbb{C}^{n}(n \geq 2)$ and let 
$\left(w, z_{j}\right)=(w, j, 0, \ldots, 0) \in \Omega, j=1, \ldots, N$. For every $t \in[0,1]$ let $\Phi_{t}$ be a biholomorphic map from $\Omega$ into $\mathbb{C}^{k} \times \mathbb{C}^{n}$, which is of the form

$$
\Phi_{t}(w, z)=\left(w, \varphi_{t}(w, z)\right), \quad z \in \mathbb{C}^{n}, w \in \mathbb{C}^{k}
$$

such that $\Phi_{t}\left(w, z_{j}\right)=\left(w, z_{j}\right) \forall w \in \mathbb{C}^{k}\left(\operatorname{resp} . \quad \Phi_{t}\left(w, z_{1}, 0, \ldots, 0\right)=\right.$ $\left.\left(w, z_{1}, 0, \ldots, 0\right) \forall z_{1} \in \mathbb{C} \forall w \in \mathbb{C}^{k}\right)$ and such that it is of class $C^{2}$ in $(t, z, w) \in[0,1] \times \Omega$. Assume that each domain $\Phi_{t}(\Omega)$ is Runge in $\mathbb{C}^{k} \times \mathbb{C}^{n}$. If $\Phi_{0}$ can be approximated on $\Omega$ by holomorphic automorphisms of $\mathbb{C}^{n}$ depending on the parameter $w \in \mathbb{C}^{k}$, fixing $\left(w, z_{j}\right)$ for every $w \in \mathbb{C}^{k}$ (resp. fixing $\left(w, z_{1}, 0, \ldots, 0\right) \forall z_{1} \in \mathbb{C} \forall w \in \mathbb{C}^{k}$ ) then for every $t \in[0,1]$ the map $\Phi_{t}$ can be approximated on $\Omega$ by holomorphic automorphisms $\alpha$ of $\mathbb{C}^{n}$ depending on the parameter $w \in \mathbb{C}^{k}$ such that $\alpha\left(w, z_{j}\right)=\left(w, z_{j}\right) \forall w \in \mathbb{C}^{k}$ (resp. $\alpha\left(w, z_{1}, 0, \ldots, 0\right)=$ $\left.\left(w, z_{1}, 0, \ldots, 0\right) \forall z_{1} \in \mathbb{C} \forall w \in \mathbb{C}^{k}\right)$.

To indicate the proof we just remark that the above theorem follows by standard techniques from the following version of the

\section{Andersén-Lempert-observation:}

Every polynomial vector field on $\mathbb{C}^{k+n}(n \geq 2)$ of the form

$$
\begin{aligned}
X=p_{1}\left(w_{1}, \ldots, w_{k}, z_{1}, z_{2}, \ldots, z_{n}\right) & \frac{\partial}{\partial z_{1}}+\ldots+ \\
& +p_{n}\left(w_{1}, \ldots, w_{k}, z_{1}, z_{2}, \ldots, z_{n}\right) \frac{\partial}{\partial z_{n}} .
\end{aligned}
$$

vanishing at the first $N$ standard points, i.e. with

$$
p_{i}\left(w, z_{j}\right)=0 \forall i=1, \ldots, n \forall j=1, \ldots, N,
$$

is a finite Lie combination of completely integrable polynomial vector fields of the above form vanishing at the first $N$ standard points. The same holds if we consider polynomial vector fields vanishing on the first coordinate line instead.

To prove this observation we develop $X$ by powers of $w$

$$
X=\sum_{\alpha} w^{\alpha} X_{\alpha}
$$

and remark that the polynomial vector fields $X_{\alpha}$ on $\mathbb{C}^{n}$ vanish at the first $N$ standard points (resp. on the first coordinate line). By Theorem 6 in [32] (the union of the first $N$ standard points is an algebraic subset of $\mathbb{C}^{n}$ of codimension at least 2) (resp. by Theorem 5.1 in [42]) they can be written as a finite Lie combination of globally integrable polynomial fields on $\mathbb{C}^{n}$ vanishing on the first $N$ standard points (resp. on the first 
coordinate line) say $\theta_{\alpha}^{i} i=1, \ldots, N(\alpha)$. The same Lie combination with $\theta_{\alpha}^{1}$ replaced by $w^{\alpha} \theta_{\alpha}^{1}$ (which is still globally integrable on $\mathbb{C}^{n+k}$, on each orbit the factor $w^{\alpha}$ is a constant) yields $w^{\alpha} X_{\alpha}$ as a Lie combination of globally integrable fields. Summing up over the multiindex $\alpha$ we get the desired result.

7.2. Families of generic finite sets with respect to affine automorphisms. As already mentioned we will use growth restrictions to prove that the embeddings for different parameters are not equivalent. The conclusion of these growth conditions will be the following: If two different embeddings in our family are equivalent, then their images can be mapped onto each other only by some affine automorphism of $\mathbb{C}^{n}$. Although it is very unlikely that the images of two different and more or less complicated embeddings can be mapped onto each other by affine automorphisms, we must be accurate in excluding this possibility. Here are some technical preparations to this point.

If we choose $n+1$ points $x_{1}, x_{2}, \ldots, x_{n+1}$ such that the difference vectors $x_{1}-x_{i}, i=2,3, \ldots, n+1$ form a basis of $\mathbb{C}^{n}$, i.e. $x_{1}, x_{2}, \ldots, x_{n+1}$ do not all lie on some affine hyperplane, then with a little linear algebra we reach the following conclusion:

Lemma 7.1. For each $m \geq n+2$ there exist $m$ points $x_{1}, x_{2}, \ldots, x_{m} \in$ $\mathbb{C}^{n}$ with the following property: No affine automorphism $\alpha \in \operatorname{Aff}\left(\mathbb{C}^{\mathrm{n}}\right)$ of $\mathbb{C}^{n}$ can map $n+2$ of them into the set $\left\{x_{1}, x_{2}, \ldots, x_{m}\right\}$.

Remark 7 . Given any open subset $\Omega$ of $\mathbb{C}^{n}$ the points $x_{1}, x_{2}, \ldots, x_{m}$ can be chosen to be contained in $\Omega$.

Let $\delta_{i j}$ denote the diagonal

$$
\delta_{i j}=\left\{\left(z_{1}, z_{2}, \ldots, z_{N}\right) \in\left(\mathbb{C}^{n}\right)^{N}: z_{i}=z_{j}\right\}
$$

and $(\underbrace{\mathbb{C}^{n} \times \cdots \times \mathbb{C}^{n}}_{N} \backslash \bigcup_{1 \leq i<j \leq N} \delta_{i j}) / S_{N}$ is the quotient (manifold since we have excluded all diagonals) by the action of the symmetric group $S_{N}$ in $N$ letters acting by permuting the entries on $N$-tuples of points in $\mathbb{C}^{n}$. The corresponding map is denoted by $\pi$.

Lemma 7.2. Let $n \geq 2$ and $k>0$ be natural numbers. Then there exists some $N \in \mathbb{N}$ such there is an injective holomorphic map

$$
\varphi: \mathbb{C}^{k} \rightarrow \underbrace{\mathbb{C}^{n} \times \cdots \times \mathbb{C}^{n}}_{N} \backslash \bigcup_{1 \leq i<j \leq N} \delta_{i j}
$$

such that the composition map

$$
\pi \circ \varphi: \mathbb{C}^{k} \rightarrow(\underbrace{\mathbb{C}^{n} \times \cdots \times \mathbb{C}^{n}}_{N} \backslash \bigcup_{1 \leq i<j \leq N} \delta_{i j}) / S_{N}
$$


is injective. Moreover if $\varphi=\left(\varphi_{1}, \varphi_{2}, \ldots, \varphi_{N}\right)$ then $\mathbb{C}^{n} \backslash \cup_{i=1}^{N} \varphi_{i}\left(\mathbb{C}^{k}\right)$ contains some nonempty open subset.

Proof. The desired number $N$ will be any number such that $n \cdot N \geq k$. Namely we will prove that there is a biholomorphic image $\Omega$ of $\left(\mathbb{C}^{n}\right)^{N}$ in $\left(\mathbb{C}^{n}\right)^{N}$ (a so called Fatou-Bieberbach domain) which does not intersect any of the diagonals $\delta_{i j}$ and so that the restriction of the quotient map $\pi:\left(\mathbb{C}^{n}\right)^{N} \rightarrow\left(\left(\mathbb{C}^{n}\right)^{N}\right) / S_{N}$ onto $\Omega$ is injective, i.e. if the point $\left(z_{1}, z_{2}, \ldots, z_{N}\right)$ is in $\Omega$ then for any permutation $\sigma \in S_{N} \backslash$ Id the point $\left(z_{\sigma(1)}, z_{\sigma(2)}, \ldots, z_{\sigma(N)}\right)$ is not contained in $\Omega$.

For this start with countably many pairwise disjoint Fatou-Bieberbach domains $\Omega_{1}, \Omega_{2}, \ldots, \Omega_{N}, \ldots$ in $\mathbb{C}^{n}$. Such domains exist, see for example [43], where countably many pairwise disjoint Fatou-Bieberbach domains are constructed arising as basins of attraction of some automorphism of $\mathbb{C}^{n}$ having countably many attracting fixed points. Now take $N$ of them and denote by $\psi_{i}$ some biholomorphic maps $\psi_{i}: \mathbb{C}^{n} \rightarrow$ $\Omega_{i} \subset \mathbb{C}^{n} \quad i=1,2, \ldots, N$.

The map

$$
\psi:\left(\mathbb{C}^{n}\right)^{N} \rightarrow\left(\mathbb{C}^{n}\right)^{N}, \psi\left(z_{1}, z_{2}, \ldots, z_{N}\right)=\left(\psi_{1}\left(z_{1}\right), \psi_{2}\left(z_{2}\right), \ldots, \psi_{N}\left(z_{N}\right)\right)
$$

is injective, its image is the Fatou-Bieberbach domain $\Omega_{1} \times \Omega_{2} \times \cdots \times$ $\Omega_{N}$ in $\left(\mathbb{C}^{n}\right)^{N}$, which does not intersect any diagonal since the $\Omega_{i}$ 's are pairwise disjoint and for the same reason $\Omega \cap \sigma(\Omega)=\emptyset$ for any permutation $\sigma \in S_{N} \backslash$ Id. The complement $\mathbb{C}^{n} \backslash \cup_{i=1}^{N}\left(\mathbb{C}^{k}\right)$ contains the union $\cup_{i=N+1}^{\infty} \Omega_{i}$ of all remaining Fatou-Bieberbach domains, hence a non-empty subset. For $n \cdot N \geq k$ we can choose some injective holomorphic map $\alpha: \mathbb{C}^{k} \mapsto\left(\mathbb{C}^{n}\right)^{N}$ and put $\varphi:=\psi \circ \alpha$, which is the desired map.

Now we are able to prove Proposition 2.1.

Proof of Proposition 2.1. By Lemma 7.2 we can find $N$ (for $n \cdot N \geq$ $k$ ) (pairwise different) points $\xi_{1}, \xi_{2}, \ldots, \xi_{N}$ in $\mathbb{C}^{n}$ parametrized by $\mathbb{C}^{k}$ such that for different parameters $w_{1} \neq w_{2} \in \mathbb{C}^{k}$ the set of points $\left\{\xi_{1}\left(w_{1}\right), \xi_{2}\left(w_{1}\right), \ldots, \xi_{m}\left(w_{1}\right)\right\}$ and $\left\{\xi_{1}\left(w_{2}\right), \xi_{2}\left(w_{2}\right), \ldots, \xi_{m}\left(w_{2}\right)\right\}$ are different. Choose $M \in \mathbb{N}$ such that $M-N \geq n+2$. By Lemma 7.1 we can find $M$ points $x_{1}, x_{2}, \ldots, x_{M}$ in $\mathbb{C}^{n}$ such that no affine automorphism except the identity can map $n+2$ of them into the set $\left\{x_{1}, x_{2}, \ldots, x_{M}\right\}$. Choose the next parametrized points $\xi_{N+1}, \xi_{N+2}, \ldots, \xi_{N+M}: \mathbb{C}^{k} \rightarrow \mathbb{C}^{n}$ to be constant

$$
\xi_{N+i}(w)=x_{i} \quad \text { for every } w \in \mathbb{C}^{k} \quad i=1,2, \ldots, M .
$$

To make the parametrized points $\xi_{1}, \xi_{2}, \ldots, \xi_{M+N}$ pairwise different (for any fixed parameter) we choose the points $x_{1}, x_{2}, \ldots, x_{M}$ from 
some open subset in the complement of all images $\xi_{j}\left(\mathbb{C}^{k}\right) \subset \mathbb{C}^{n} j=$ $1,2, \ldots, N$.

We claim that $\left\{\xi_{1}(w), \xi_{2}(w), \ldots, \xi_{N+M}(w)\right\}$ satisfy our condition (so $m=N+M)$. Indeed suppose that for two different parameters $w_{1} \neq w_{2} \in \mathbb{C}^{k}$ there is an affine automorphism $\alpha \in \operatorname{Aff}\left(\mathbb{C}^{n}\right)$ which map the set of points $\left\{\xi_{1}\left(w_{1}\right), \xi_{2}\left(w_{1}\right), \ldots, \xi_{N+M}\left(w_{1}\right)\right\}$ onto the set of points $\left\{\xi_{1}\left(w_{2}\right), \xi_{2}\left(w_{2}\right), \ldots, \xi_{N+M}\left(w_{2}\right)\right\}$. Since $M-N \geq n+2$ at least $n+2$ of the last $M$ points, $\left(x_{1}, x_{2}, \ldots, x_{M}\right)$, are mapped by $\alpha$ into $\left\{x_{1}, x_{2}, \ldots, x_{M}\right\}$, and note that at most $N$ points among them can be mapped onto the first $N$ points! By the choice of $x_{1}, x_{2}, \ldots, x_{M}$ according to Lemma 7.1 this implies that $\alpha$ is the identity map. But this means that the identity maps the last $M$ (constant) points onto themselves, hence the points $\left\{\xi_{1}\left(w_{1}\right), \xi_{2}\left(w_{1}\right), \ldots, \xi_{N}\left(w_{1}\right)\right\}$ onto the points $\left\{\xi_{1}\left(w_{2}\right), \xi_{2}\left(w_{2}\right), \ldots, \xi_{N}\left(w_{2}\right)\right\}$, which is impossible since those sets are different by Lemma 7.2. Thus no such affine automorphism $\alpha$ exists.

7.3. Moving finitely many parametrized points. Recall that we needed the notion of simultaneously standardazable points in the proof of the main theorem. Given $N$ parametrized points $\zeta_{i}: \mathbb{C}^{k} \rightarrow C^{n}$. If we can find an automorphism $\psi \in \mathrm{Aut}_{h o l}^{k}\left(\mathbb{C}^{n}\right)$ such that

$$
\psi\left(w, \zeta_{i}(w)\right)=(w,(i, 0)) \text { for all } i=1,2, \ldots, N \text { and for all } w \in \mathbb{C}^{k},
$$

we say that the points are simultaneously standardazable.

The following theorem is a special case of the Oka-Grauert-Gromov$\mathrm{h}$ principle in complex analysis. Even if our application would fit in the classical context proved by Grauert [23], 24], [25] (our transition functions are affine linear, i.e. contained in a complex Lie group) we formulate it in a more general (but not too general in order to avoid the discussion of sprays) way. For reference see [15], section 2.3 in [26] or Theorem 1.4 in [18.

Theorem 7.2 (Oka principle with approximation). Let $X$ be a Stein manifold and let $Z$ be a locally trivial bundle such that the fiber $Z_{x}$ is isomorphic to $\mathbb{C}^{n}$. If $s: X \rightarrow Z$ is a continuous section which is holomorphic in a neighborhood of an $\mathcal{O}(X)$-convex compact subset $K$ then there exists a holomorphic section $\tilde{s}: X \rightarrow Z$ such that $\tilde{s}$ approximates s uniformly on $K$.

Example 2. Let $q_{1}(w), q_{2}(w), \ldots, q_{n}(w): \mathbb{C}^{k} \rightarrow \mathbb{C}$ be holomorphic functions without common zeros. We want to find holomorphic functions $h_{1}(w), h_{2}(w), \ldots, h_{n}(w): \mathbb{C}^{k} \rightarrow \mathbb{C}$ such that

$$
h_{1}(w) q_{1}(w)+h_{2}(w) q_{2}(w)+\ldots+h_{n}(w) q_{n}(w)=1
$$


for every $w \in \mathbb{C}^{k}$, i.e. find a point $\left(h_{1}(w), h_{2}(w), \ldots, h_{n}(w)\right)$ in the hyperplane given by

$$
x_{1} q_{1}(w)+x_{2} q_{2}(w)+\ldots+x_{n} q_{n}(w)=1 .
$$

Since $q_{1}(w), q_{2}(w), \ldots, q_{n}(w)$ do not have common zeros, we can find local solutions: If (e.g.) $q_{1}(w) \neq 0$ then we can solve the problem in a neighborhood of $w_{0}$ by setting $h_{2}(w)=\ldots=h_{n}(w)=1$ and

$$
h_{1}(w)=\frac{1-\left(h_{2}(w) q_{2}(w)+\ldots+h_{n}(w) q_{n}(w)\right)}{q_{1}(w)} .
$$

Let $Z$ be the (locally trivial with affine linear transition functions) bundle over $\mathbb{C}^{k}$ such that the fiber $Z_{w}$ is the hyperplane (8) in $\mathbb{C}^{n}$. Given a holomorphically convex compact set $J \in \mathbb{C}^{k}$ and a holomorphic section $s$ of the bundle over a neighborhood of $J$ we can, by standard arguments in obstruction theory (all homotopy groups of the fiber vanish), extend it to a continuous section $s: \mathbb{C}^{k} \rightarrow Z$. Theorem 7.2 gives a holomorphic section $\tilde{s}: \mathbb{C}^{k} \rightarrow Z$ which approximates $s$ uniformly on the compact $J$.

Lemma 7.3. Given a holomorphic map $\xi=\left(\xi_{1}, \ldots, \xi_{n}\right): \mathbb{C}^{k} \rightarrow \mathbb{C}^{n}$, $(n \geq 2)$, always disjoint from the first $N$ standard points, $\xi(w) \notin$ $\cup_{i=1}^{N}\{(i, 0, \ldots, 0)\}$, and such that the functions $\xi_{2}, \ldots, \xi_{n} \in \mathcal{O}\left(\mathbb{C}^{k}\right)$ have no common zero on $\mathbb{C}^{k}$. Then there exist $\alpha \in \mathrm{Aut}_{\mathrm{hol}}^{\mathrm{k}}\left(\mathbb{C}^{\mathrm{n}}\right)$ fixing the first $N$ standard points, $\alpha(w, i, 0, \ldots, 0)=(w, i, 0, \ldots, 0)$ with $\alpha(w, \xi(w))=$ $\left(w, z_{0}\right)$, i.e. $\alpha(w, \xi(w))$ is a constant point $\left(w, z_{0}\right)$ for every $w \in \mathbb{C}^{k}$.

Moreover given a ball $J=r \overline{\mathbb{B}}_{k} \subset \mathbb{C}^{k}$ and a number $R>N$ such that for $w \in J$ hold: $|\xi(w)|>R$. Then for any $\epsilon>0$ the automorphism $\alpha$ can be chosen in such a way that

$$
\max _{w \in J,|z| \leq R}|\alpha(w, z)-(w, z)|<\epsilon .
$$

Proof. The first step consists in an application of Theorem 7.1 with fixing the first coordinate line to bring the points $\xi(w), w \in J$, arbitrarily nearby to a constant position. To apply the theorem let $\Omega=\bigcup_{w \in r^{\prime} \mathbb{B}_{k}}\{w\} \times\left\{R^{\prime} \mathbb{B}_{n} \cup \epsilon_{1} \mathbb{B}_{n}(\xi(w))\right\}$, where $\mathbb{B}_{n}(\xi(w))$ is the unit ball in $\mathbb{C}^{n}$ with center in $\xi(w)$, with $r^{\prime}, R^{\prime}$ slightly bigger than $r, R$ and $\epsilon_{1}$ sufficiently small so that $\epsilon_{1} \mathbb{B}_{n}(\xi(w))$ has empty intersection with the first coordinate line for all $w \in r^{\prime} \mathbb{B}_{k}$. Note that $\Omega$ is Runge in $\mathbb{C}^{k} \times \mathbb{C}^{n}$.

Approximating the map $\Phi_{t}:[0,1] \times \Omega \rightarrow \mathbb{C}^{k} \times \mathbb{C}^{n}$ defined by $\Phi_{t}(w, z)=(w, z)$ for every $w \in r^{\prime} \mathbb{B}_{k}, z \in R^{\prime} \mathbb{B}_{n}$ and $\Phi_{t}(w, z)=$ $(w, \xi((1-t) w)+z-\xi(w))$ for $w \in r^{\prime} \mathbb{B}_{k}, z \in \epsilon_{1} \mathbb{B}_{n}(\xi(w))$, gives an 


$$
\begin{aligned}
& \alpha_{1} \in \mathrm{Aut}_{\mathrm{hol}}^{\mathrm{k}}\left(\mathbb{C}^{\mathrm{n}}\right): \\
& \begin{array}{r}
\alpha_{1}\left(w,\left(z_{1}, 0, \ldots, 0\right)\right)=\left(w,\left(z_{1}, 0, \ldots, 0\right)\right) \forall z_{1} \in \mathbb{C} \forall w \in \mathbb{C}^{k} \\
\left|\alpha_{1}(w, z)-(w, z)\right|_{w \in J, z \in R \mathbb{B}_{n}}<\epsilon \\
\left|\alpha_{1}(w, \xi(w))-(w, \xi(0))\right|<\epsilon \quad \forall w \in J
\end{array}
\end{aligned}
$$

where $\epsilon$ is arbitrarily small.

Remark that the last $n-1$ coordinate functions of $\alpha_{1}(w, \xi(w))$ have no common zero on $\mathbb{C}^{k}$, since by assumption the same was true for $\xi(w)$ and the first coordinate line is fixed by $\alpha_{1}$.

A second application of Theorem 7.1 again with fixing the first coordinate line using a $C^{2}$-path from $\xi(0)$ to the point $(2 R, 1,0, \ldots, 0)$ not intersecting the first coordinate line and not intersecting $R^{\prime} \mathbb{B}_{n}$ shows that we in addition can assume

$$
\left|\alpha_{1}(w, \xi(w))-(2 R, 1,0, \ldots, 0)\right|<\epsilon
$$

for $w \in J$.

Denote the coordinate functions of $\alpha_{1}(w, \xi(w))$ by $\left(\check{q}_{1}(w), \check{q}_{2}(w), \ldots\right.$, $\left.\check{q}_{n}(w)\right)$ and observe that $\check{q}_{2}(w), \ldots, \check{q}_{n}(w)$ have no common zeros for $w \in \mathbb{C}^{k}$.

Now define functions $\hat{h}_{i} \in \mathcal{O}(J)$ by

$$
\hat{h}_{3}(w)=\ldots=\hat{h}_{n}(w)=1
$$

and

$$
\hat{h}_{2}(w)=\frac{1-\check{q}_{3}(w) \hat{h}_{3}(w)-\ldots-\check{q}_{n}(w) \hat{h}_{n}(w)}{\check{q}_{2}(w)}
$$

for $w \in J$. Note that $\hat{h}_{2}(w) \approx 1$ for $w \in J$.

By Theorem 7.2 (see example thereafter) we have that for every $\epsilon>0$ there exist $h_{i} \in \mathcal{O}\left(\mathbb{C}^{k}\right)$ with

$$
\sum_{i=2}^{n} h_{i}(w) \check{q}_{i}(w)=1, \forall w \in \mathbb{C}^{k}
$$

and

$$
\left\|h_{i}(w)-\hat{h}_{i}(w)\right\|_{J}<\epsilon
$$

which implies $h_{i}(w) \approx 1$ for $w \in J$ for all $i=2, \ldots, n$. Define an automorphism $\alpha_{2} \in \mathrm{Aut}_{\mathrm{hol}}^{\mathrm{k}}\left(\mathbb{C}^{\mathrm{n}}\right)$ by

$$
\begin{aligned}
&(w, z) \mapsto \\
&\left(w, z_{1}+\left(2 R-\check{q}_{1}(w)\right)\left[z_{2} h_{2}(w)+z_{3} h_{3}(w)+\ldots z_{n} h_{n}(w)\right], z_{2}, z_{3}, \ldots, z_{n}\right) .
\end{aligned}
$$

It holds:

$$
\alpha_{2}(w, \check{q}(w))=\left(w, 2 R, \check{q}_{2}(w), \ldots, \check{q}_{n}(w)\right)
$$


and

$$
\alpha_{2}(w,(i, 0, \ldots, 0))=(w,(i, 0, \ldots, 0))
$$

The next step is to construct an automorphism $\alpha_{3} \in \mathrm{Aut}_{\mathrm{hol}}^{\mathrm{k}}\left(\mathbb{C}^{\mathrm{n}}\right)$ that moves $\alpha_{2}(w, \breve{q}(w))$ to $(w, 2 M, 0, \ldots, 0)$. So define a polynomial on $\mathbb{C}$

$$
Q(t)=\left((t-1)(t-2) \cdots(t-N) \frac{1}{(2 R-1)(2 R-2) \cdots(2 R-N)}\right)^{H}
$$

where $H$ is so large that $|t|<R$ implies $|Q(t)|<\epsilon$, and define the automorphism $\alpha_{3}$ by

$$
\begin{aligned}
& (w, z) \mapsto \\
& \quad\left(w, z_{1}, z_{2}-Q\left(z_{1}\right) \check{q}_{2}(w), z_{3}-Q\left(z_{1}\right) \check{q}_{3}(w), \ldots, z_{n}-Q\left(z_{1}\right) \check{q}_{n}(w)\right) .
\end{aligned}
$$

From this we get

$$
\alpha_{3}(w, i, 0, \ldots, 0)=(w, i, 0, \ldots, 0)
$$

for $i=1, \ldots N$,

$$
\alpha_{3} \circ \alpha_{2}(w, \check{q}(w))=(w, 2 R, 0, \ldots, 0)
$$

and it is easy to check that

$$
\max _{w \in J,|z| \leq R}\left|\alpha_{3} \circ \alpha_{2} \circ \alpha_{1}(w, z)\right|
$$

is arbitrarily small. The composition $\alpha=\alpha_{3} \circ \alpha_{2} \circ \alpha_{1}$ is our desired automorphism.

Remark 8. The reason that we bring the points $\{\xi(w), w \in J\}$ first near to $(2 R, 1,0, \ldots, 0)$ (instead of $(2 R, 0, \ldots, 0)$ directly) and make them afterwards constant at the point $(2 R, 0, \ldots, 0)$ is the following: Our method could lead to a big movement of $R \mathbb{B}_{n}$, as we see in the following example.

Example 3. Let $K=K_{1} \times \bar{\Delta}^{n}$ where $\bar{\Delta}^{n}$ is the closure of the unit polydisc in $\mathbb{C}^{n}$ and $K_{1}$ some compact set in the parameter space $\mathbb{C}^{k}$. We will consider the following perturbation of the first $N$ standard points:

We suppose that the first $N-1$ points remains at their standard positions,

$$
\zeta_{i}(w)=(i, 0, \ldots, 0) \quad i=1,2, \ldots, N-1
$$

and the $N$-th point is moved by some very small amount from the standard position

$$
\zeta_{n}(w)=\left(2 R-\epsilon, \epsilon^{2}, \ldots, \epsilon^{2}\right) .
$$

According to the proof of Lemma 7.3 we have to find holomorphic functions $h_{2}(w), h_{3}(w), \ldots, h_{n}(w)$ with $\sum_{i=2}^{N} h_{i}(w) \epsilon^{2}=-2 R+\epsilon+2 R=$ 
$\epsilon$, i.e. $\sum_{i=2}^{N} h_{i}(w)=1 / \epsilon$. Therefore the automorphism $\alpha_{2} \in \operatorname{Aut}_{h o l}^{k}\left(\mathbb{C}^{n}\right)$ defined by

$$
\left(w, z_{1}, z_{2}, \ldots, z_{n}\right) \mapsto\left(w, z_{1}+z_{2} h_{2}(w)+\ldots+z_{n} h_{n}(w), z_{2}, z_{3}, \ldots, z_{n}\right)
$$

moves for instance all points of the form $\left(0, z_{1}, 1, \ldots, 1\right) \in K_{1} \times \bar{\Delta}^{n}$ by the vector $(0,1 / \epsilon, 0, \ldots, 0)$, i.e. $\left(0, z_{1}, 1, \ldots, 1\right) \mapsto\left(0, z_{1}+1 / \epsilon, 1, \ldots, 1\right)$, which has length going to infinity when our perturbation of the $N$-th point is going to zero.

From Lemma 7.3 we get Proposition 2.2

Proof of Proposition 2.2. Proceed by induction over the number $N$ of points: For $N=1$ the (parametrized translation) automorphism defined by $(w, z) \mapsto\left(w, z-\zeta_{1}(w)+1\right)$ solves the problem in general, i.e. without any assumption on the dimension $k$ of the parameter space.

Suppose the problem is solved for $N-1$ parametrized points. To solve it for $N$ points take by induction assumption an automorphism $\alpha_{1} \in \operatorname{Aut}_{h o l}^{k}\left(\mathbb{C}^{n}\right)$ which moves the first $N-1$ points to their standard places

$\alpha_{1}\left(w, \zeta_{i}(w)\right)=(w,(i, 0))$ for all $i=1,2, \ldots, N-1$ and for all $w \in \mathbb{C}^{k}$.

It is not difficult to find an automorphism $\alpha_{2} \in \operatorname{Aut}_{h o l}^{k}\left(\mathbb{C}^{n}\right)$ which fixes the first $N-1$ standard points, $\alpha_{2}(w,(i, 0))=(w,(i, 0))$ for all $i=1,2, \ldots, N-1$ and for all $w \in \mathbb{C}^{k}$, such that the submanifold of $\mathbb{C}^{k+n}$ described by the (moved) last parametrized point

$$
U:=\left\{\left(w, \alpha_{2} \circ \alpha_{1} \circ \zeta_{N}(w)\right): w \in \mathbb{C}^{k}\right\} \subset \mathbb{C}^{k+n}
$$

is transversal to the (parametrized) $z_{1}$-axis

$$
V:=\left\{\left(w,\left(z_{1}, z_{2}, \ldots, z_{n}\right)\right) \in \mathbb{C}^{k+n}: z_{2}=z_{3}=\ldots=z_{n}=0\right\} .
$$

Because of the dimension assumption this means that the two submanifolds $U$ and $V$ do not meet $(\operatorname{dim} U+\operatorname{dim} V=k+(k+1)<n+k=$ $\left.\operatorname{dim} \mathbb{C}^{k+n}\right)$. In other words we are in the position of Lemma 7.3 and find an automorphism $\alpha_{3} \in \operatorname{Aut}_{h o l}^{k}\left(\mathbb{C}^{n}\right)$ fixing the first $N-1$ standard points and moving $\left(w, \alpha_{2} \circ \alpha_{1} \circ \zeta_{N}(w)\right)$ to its standard place. The composition $\alpha_{3} \circ \alpha_{2} \circ \alpha_{1}$ is the desired automorphism moving all $N$ parametrized points into their standard positions.

We are now set to prove the interpolation lemma, Lemma 2.3.

proof of Lemma 2.3. Since by the dimension assumption $\operatorname{dim} X+k<n$ the points $b_{i}(w)$ are simultaneously standardizable(see Corollary 2.2), we can find $\alpha_{1} \in \operatorname{Aut}_{\mathrm{hol}}^{\mathrm{k}}\left(\mathbb{C}^{\mathrm{n}}\right)$ such that $\alpha_{1}\left(w, b_{i}(w)\right)=(w,(i, 0, \ldots, 0))$ for every $w \in \mathbb{C}^{k}$. Choose $R>0$ such that $\pi_{2}\left(\alpha_{1}(K)\right) \subset R \mathbb{B}_{n}$ and 
choose $M$ such that $\alpha_{1}^{-1}\left(R \mathbb{B}_{n}\right) \subset M \mathbb{B}_{n}$. Let $C$ be a positive real constant such that the point $\tilde{p}(w)=C p(w)$ is outside of the ball of radius $M$ for every $w \in \bar{\Delta}$. By transversality and our dimension condition (1) we can assume that an arbitrary small perturbation $\gamma_{p}$ of the path $p(w)+t(\tilde{p}(w)-p(w)), t \in[0,1]$, does not intersect $\pi_{2}\left(\phi\left(\bar{\Delta} \times \bar{X}_{R}\right)\right)$ for any $w \in \bar{\Delta}$. Construct $\tilde{q}(w)$ and a path $\gamma_{q}$, in the same way with the additional demand that the path for $q$ does not intersect the path for $p$.

By the Andersén-Lempert-theorem with parameters and fixing standard points (see Theorem [7.1) applied to the set $\alpha_{1}(K)$ union with a neighborhood of the paths $\alpha_{1}\left(\gamma_{p}\right)$ and $\alpha_{1}\left(\gamma_{q}\right)$ there exists an $\alpha_{2} \in$ Aut $\mathrm{t}_{\mathrm{hol}}^{\mathrm{k}}\left(\mathbb{C}^{\mathrm{n}}\right)$ such that $\alpha_{2}(w, z)$ is close to the identity for $(w, z) \in K$ and $\left|\pi_{2}\left(\alpha_{2} \circ \alpha_{1}(w, q(w))\right)\right|>R$ for every $w \in \bar{\Delta}$. Furthermore we have $\alpha_{2} \circ \alpha_{1}\left(w, b_{i}(w)\right)=(w,(i, 0, \ldots, 0))$ for every $w \in \mathbb{C}^{k}$. Moreover $\alpha_{2} \circ \alpha_{1}(K) \subset R \mathbb{B}_{n}$. To be able to apply Lemma 7.3 we perturb $\alpha_{2} \circ \alpha_{1}(w, p(w))$ slightly to make sure that the last $n-1$ coordinate functions have no common zero on $\mathbb{C}^{k}$, at the same time fixing the points $(w,(i, 0, \ldots, 0)), i=1, \ldots, N$. This is possible by transversality and our dimension assumption (11), i.e. $\operatorname{dim} X+k<n$. Now an application of Lemma 7.3 gives an automorphism $\alpha_{3}$ arbitrarily close to identity on $\bar{\Delta} \times R \mathbb{B}_{n}$ fixing the first $N$ standard points, such that $\alpha_{3} \circ \alpha_{2} \circ \alpha_{1}(w, p(w))=(w, 2 R, 1,0, \ldots, 0)$ for every $w \in \mathbb{C}^{k}$.

By another application of the same lemma, we can in addition assume that $\alpha_{3} \circ \alpha_{2} \circ \alpha_{1}(w, q(w))=(w, 2 R, 2,0, \ldots, 0)$ for every $w \in \mathbb{C}^{k}$.

Finally we take a polynomial $\tilde{P}\left(z_{1}\right)$ such that $\tilde{P}(2 R)=1,\left|\tilde{P}\left(z_{1}\right)\right|<\epsilon$ for $z_{1} \in(R+1) \overline{\mathbb{B}}_{1}$ and $\tilde{P}\left(z_{1}\right)=0$ to order $l$, for $z_{1} \in\{1, \ldots N\}$. Then define the automorphism

$$
\alpha_{4}(w, z)=\left(w, z_{1}, z_{2}+\tilde{P}\left(z_{1}\right), z_{3}, \ldots, z_{n}\right) .
$$

Consequently

$$
\alpha_{4}(w, i, 0, \ldots, 0)=(w, i, 0, \ldots, 0)
$$

to prescribed order for $i=1, \ldots N$ and $\alpha_{4} \circ \alpha_{3} \circ \alpha_{2} \circ \alpha_{1}(w, p(w))=$ $\alpha_{3} \circ \alpha_{2} \circ \alpha_{1}(w, q(w))$.

In total, the automorphism

$$
\alpha=\alpha_{1}^{-1} \circ \alpha_{2}^{-1} \circ \alpha_{3}^{-1} \circ \alpha_{4} \circ \alpha_{3} \circ \alpha_{2} \circ \alpha_{1},
$$

will have the properties stated in the lemma.

7.4. Proof of osculation lemma. Remember that we will mark a finite number of points. The points will be used to exclude affine automorphisms in the main theorem. 
Before we come to this point, a standard jet interpolation lemma in a parametrized form is established. This form is quite easy, since we do assume that the linear part of each prescribed jet is the identity. For general linear parts one would need (in order to get the linear part from shears) to write a holomorphic (depending on the parameter in $\left.\mathbb{C}^{k}\right)$ invertible matrix as a product of holomorphic elementary matrices. This is the so called Vaserstein problem posed by Gromov in [26]. Although it was recently solved by Ivarsson and the first author [29], [30] we will restrict ourselves to the present simple version of our lemma since it is fully sufficient for the purpose of the present paper.

Lemma 7.4. Let $\xi_{i}=(i, i, \ldots, i) \in \mathbb{C}^{n}$ for $i=0,1,2, \ldots, N$ and let $P_{i}: \mathbb{C}^{k} \times \mathbb{C}^{n} \rightarrow \mathbb{C}^{n}$ be polynomial maps of degree $s$ such that

$$
P_{i}(w, z)=\xi_{i}+\left(z-\xi_{i}\right)+O\left(\left|z-\xi_{i}\right|^{2}\right)
$$

for $z \rightarrow \xi_{i}$ and all $w \in \mathbb{C}^{k}$.

Then there exists $\kappa \in \mathrm{Aut}_{\mathrm{hol}}^{\mathrm{k}}\left(\mathbb{C}^{\mathrm{n}}\right)$ such that

$$
\pi_{2}\left(\kappa(w, z)-\left(w, P_{i}(w, z)\right)\right)=O\left(\left|z-\xi_{i}\right|^{s+1}\right)
$$

for $z \rightarrow \xi_{i}$ with $i=0,1,2, \ldots, N$.

Remark 9. The reason that we have chosen $\xi_{i}=(i, i, \ldots, i) \in \mathbb{C}^{n}$ for $i=0,1,2, \ldots, N$ is to ensure that our points have pairwise different projections along all coordinate directions. This is suitable for the use of shears.

Proof. The proof goes exactly as in the non-parametric case (see Step 2.10 in Forstneric [14]) by induction over the number of points and the order of the jets. The beginning step of the induction (first order) is empty in our case. To realize the homogeneous part $P^{j}$ of order $j \geq 2$ of a polynomial map $P$ by a composition of overshears on $\mathbb{C}^{n}$ depending holomorphically on the parameter on $w$ we need to establish the existence of finitely many linear functionals $\lambda_{i}$ together with vectors $v_{i}$ having the properties $\lambda_{i}\left(v_{i}\right)=0$ and $\left|v_{i}\right|=1$ such that

$$
\begin{aligned}
P^{j}(w, z) & =\sum_{i} c_{i}(w)\left(\lambda_{i}\left(z-\xi_{1}\right)\right)^{j} v_{i}+ \\
& +\sum_{k} d_{k}(w)\left(\lambda_{k}\left(z-\xi_{1}\right)\right)^{j-1}\left\langle z-\xi_{1}, v_{k}\right\rangle v_{k}
\end{aligned}
$$

with holomorphic functions $c_{i}, d_{i} \in \mathcal{O}\left(\mathbb{C}^{k}\right)$.

This follows from the purely algebraic fact, Lemma 7.6 below. 
We denote the complex vector space $\mathbb{C}^{n}$ by $V, S^{k}\left(V^{*}\right)$ denotes the vector space of homogenous polynomials of degree $k$ on $V$ and $r_{k, n}=$ $\left(\begin{array}{c}n+k-1 \\ n-1\end{array}\right)$ its dimension.

Lemma 7.5. There exist $r_{k, n}$ linear forms $\lambda_{1}, \lambda_{2}, \ldots, \lambda_{r_{k, n}} \in\left(\mathbb{C}^{n}\right)^{*}$ such that the homogenous polynomials $\left(\lambda_{i}\right)^{k}$ of degree $k i=1,2, \ldots, r_{k, n}$ form a basis of $S^{k}\left(V^{*}\right)$. Moreover the $\lambda_{i}$ can be chosen from any nonempty open subset $\Omega$ of $\left(\mathbb{C}^{n}\right)^{*}$.

Proof. Take any nonzero element $\lambda_{0} \in \Omega \in V^{*}$. The map $V^{*} \rightarrow S^{k}\left(V^{*}\right)$ defined by $\lambda \mapsto \lambda^{k}$ is $\mathrm{Gl}(\mathrm{V})$-equivariant and since $S^{k}\left(V^{*}\right)$ is an irreducible $\mathrm{Gl}(\mathrm{V})$-module the linear span of the $\mathrm{Gl}(\mathrm{V})$-orbit through $\lambda_{0}$

$$
\operatorname{span}\left\{\left(g \cdot \lambda_{0}\right)^{k}, \quad g \in \mathrm{Gl}(\mathrm{V})\right\}
$$

is the whole module $S^{k}\left(V^{*}\right)$. The same holds for any open part of the orbit, i.e.

$$
\operatorname{span}\left\{\left(g \cdot \lambda_{0}\right)^{k}, \quad g \in U\right\}=S^{k}\left(V^{*}\right)
$$

for any open subset $U$ of $\mathrm{Gl}(\mathrm{V})$, since if the left hand side would be contained in some proper linear subspace $W \subset S^{k}\left(V^{*}\right)$ then by the identity theorem for holomorphic mappings the whole orbit would be contained in $W$ contradicting the irreducibility of $S^{k}\left(V^{*}\right)$. We can therefore find $r_{k, n}$ group elements $g_{1}, g_{2}, \ldots, g_{r_{k, n}} \in \mathrm{Gl}(\mathrm{V})$ contained in some open neighborhood $U$ of the identity (with $U \cdot \lambda_{0} \subset \Omega$ ) such that the homogenous polynomials $\left(g_{j} \cdot \lambda_{0}\right)^{k} k=1,2, \ldots, r_{k, n}$ form a basis of $S^{k}\left(V^{*}\right)$.

Lemma 7.6. There exist $n \cdot\left(\begin{array}{c}n+k-2 \\ n-1\end{array}\right)-\left(\begin{array}{c}n+k-2 \\ n-1\end{array}\right)$ linear forms $\lambda_{i} \in\left(\mathbb{C}^{n}\right)^{*}$ and vectors $v_{i} \in \mathbb{C}^{n}$ with $\lambda_{i}\left(v_{i}\right)=0$ and $\left\|v_{i}\right\|=1 i=1,2, \ldots,\left(\begin{array}{c}n+k-2 \\ n-1\end{array}\right)$ together with $\left(\begin{array}{c}n+k-2 \\ n-1\end{array}\right)$ linear forms $\tilde{\lambda}_{j} \in\left(\mathbb{C}^{n}\right)^{*}$ and vectors $w_{j} \in \mathbb{C}^{n}$ with $\lambda_{j}\left(w_{j}\right)=0$ and $\left\|w_{j}\right\|=1 j=1,2, \ldots, n \cdot\left(\begin{array}{c}n+k-2 \\ n-1\end{array}\right)-\left(\begin{array}{c}n+k-2 \\ n-1\end{array}\right)$ such that the homogenous polynomial maps

$$
z \mapsto\left(\lambda_{i}(z)\right)^{k} v_{i}, \quad i=1,2, \ldots, n \cdot\left(\begin{array}{c}
n+k-1 \\
n-1
\end{array}\right)-\left(\begin{array}{c}
n+k-2 \\
n-1
\end{array}\right)
$$

of degree $k$ together with the homogenous polynomial maps

$$
z \mapsto\left(\tilde{\lambda}_{j}(z)\right)^{k-1}\left\langle z, w_{j}\right\rangle w_{j}, \quad j=1,2, \ldots,\left(\begin{array}{c}
n+k-2 \\
n-1
\end{array}\right)
$$

of degree $k$ form a basis of the vector space $V_{k} \cong S^{k}\left(\left(\mathbb{C}^{n}\right)^{*}\right) \otimes \mathbb{C}^{n}$ of homogenous polynomial maps of degree $k$. Moreover if $v_{0} \in \mathbb{C}^{n}$ and a non-zero functional $\lambda_{0} \in\left(\mathbb{C}^{n}\right)^{*}$ with $\lambda_{0}\left(v_{0}\right)=0$ and $\left\|v_{0}\right\|=1$ and a number $\epsilon>0$ are given, then the vectors $v_{i}, w_{j}$ together with the 
functionals $\lambda_{i}, \tilde{\lambda}_{j}$ can be chosen with $\left\|v_{0}-v_{i}\right\|<\epsilon\left\|v_{0}-w_{j}\right\|<\epsilon$ and $\left\|\lambda_{0}-\lambda_{i}\right\|<\epsilon,\left\|\lambda_{0}-\tilde{\lambda}_{j}\right\|<\epsilon$.

Proof. Set $V=\mathbb{C}^{n}$. The vector space $V_{k} \cong S^{k}\left(V^{*}\right) \otimes V$ is as $\mathrm{Gl}(\mathrm{V})$ module isomorphic to the direct sum of two irreducible representations $W_{1} \oplus W_{2}$, where $W_{1}$ is isomorphic to $S^{k-1}\left(V^{*}\right)$ and $W_{2}$ is isomorphic to the kernel of the $\mathrm{Gl}(\mathrm{V})$-equivariant map

$$
\psi: S^{k}\left(V^{*}\right) \otimes V \rightarrow S^{k-1}\left(V^{*}\right), \quad X \mapsto \operatorname{div} X .
$$

We will provide some "section" of $\psi$ : By Lemma 7.5 there exist $\tilde{\lambda}_{j} \in V^{*} j=1,2, \ldots,\left(\begin{array}{c}n+k-2 \\ n-1\end{array}\right)\left(\epsilon\right.$-near to $\lambda_{0}$ if desired $)$ such that the homogenous polynomials $\tilde{\lambda}_{i}^{k-1}$ form a basis of $S^{k-1}\left(V^{*}\right)$. Choose vectors $w_{j}$ with $\tilde{\lambda}_{j}\left(w_{j}\right)=0$ and $\left\|w_{j}\right\|=1$ (and $\epsilon$-near to $v_{0}$ if desired). For a homogenous polynomial $p(z)$ of degree $k-1$ we write it in the basis $p(z)=\sum_{j=1}^{\left(\begin{array}{c}n+k-2 \\ n-1\end{array}\right)} d_{j}\left(\tilde{\lambda}_{j}(z)\right)^{k-1}$ and define the section $s(p)$ by

$$
s(p)(z)=\sum_{j=1}^{\left(\begin{array}{c}
n+k-2 \\
n-1
\end{array}\right)} d_{j}\left(\tilde{\lambda}_{j}(z)\right)^{k-1}\left\langle z, w_{j}\right\rangle w_{j} .
$$

An easy calculation shows $\psi(s(p)))=\operatorname{div} s(p)=p$. Thus the homogenous polynomial maps $z \mapsto\left(\tilde{\lambda}_{j}(z)\right)^{k-1}\left\langle z, w_{j}\right\rangle w_{j} j=1,2, \ldots,\left(\begin{array}{c}n+k-2 \\ n-1\end{array}\right)$ form a basis of some linear subspace of $S^{k}\left(V^{*}\right) \otimes V$ complementary to the kernel of $\psi$. Now take our nonzero linear functional $\lambda_{0} \in V^{*}$ and some vector $v_{0} \in V$ with $\lambda_{0}\left(v_{0}\right)=0$ and $\left\|v_{0}\right\|=1$. Since $\operatorname{ker} \psi \cong W_{2}$ is an irreducible $\mathrm{Gl}(\mathrm{V})$-module the linear span of any $\mathrm{Gl}(\mathrm{V})$-orbit through a nonzero point in $W_{2}$ is the whole vector space $W_{2} \cong \operatorname{ker} \psi$. Since $\lambda_{0}\left(v_{0}\right)=0$ the element $\lambda_{0}^{k} \otimes v_{0}$ is such a point and like in the proof of Lemma 7.5 we find group elements $g_{1}, g_{2}, \ldots, g_{n \cdot\left(\begin{array}{c}n+k-1 \\ n-1\end{array}\right)-\left(\begin{array}{c}n+k-2 \\ n-1\end{array}\right)} \in \mathrm{Gl}(\mathrm{V})$ contained in any given nonempty open neighborhood of the identity element such that the homogenous polynomial maps $g_{j} \cdot\left(\lambda_{0}^{k} \otimes v_{0}\right)=$ $\left(g_{j} \cdot \lambda_{0}\right) g_{j} \cdot v_{0}$ form a basis of ker $\psi$. Defining

lambda $a_{i}=g_{i} \cdot \lambda_{0}\left(\right.$ remember $\left.g \cdot \lambda_{0}(v):=\lambda_{0}\left(g^{-1} v\right)\right)$ and $\tilde{v}_{i}=g_{i} \cdot v_{0}, v_{i}=$ $\frac{\tilde{v}_{i}}{\left|\tilde{v}_{i}\right|}$ (instead of normalizing we could have chosen the $g_{j}$ from the unitary group since by the identity principle in complex analysis any irreducible $\mathrm{Gl}_{\mathrm{n}}$-representation is $\mathrm{U}_{\mathrm{n}}$-invariant) we get a basis $\lambda_{i}^{k} \otimes v_{i}$ $i=1,2, \ldots, n \cdot\left(\begin{array}{c}n+k-1 \\ n-1\end{array}\right)-\left(\begin{array}{c}n+k-2 \\ n-1\end{array}\right)$ of $\operatorname{ker} \psi$. Together with the above constructed basis of the complementary subspace it forms a basis of the vector space $V_{k} \cong S^{k}\left(\left(\mathbb{C}^{n}\right)^{*}\right) \otimes \mathbb{C}^{n}$ of homogenous polynomial maps of degree $k$.

Using these prerequisits we give the proof of Lemma 2.4 
proof of Lemma 2.4. Since the points $\xi_{1}(w), \ldots, \xi_{t}(w)$ are simultaneously standardizable we can assume that $\xi_{i}(t)=(i, i, \ldots, i)$ for $1 \leq$ $i \leq t$. Now we want to apply Lemma 7.4 to make $\kappa(M(w))$ osculating of order $l$. This means we have to ensure that there is for each $i$ a holomorphically depending on $w \in \mathbb{C}^{k}$ polynomial map $P_{i}(w, z)$ with

$$
P_{i}(w, z)=\xi_{i}+\left(z-\xi_{i}\right)+O\left(\left|z-\xi_{i}\right|^{2}\right)
$$

We then get osculation of order $l$ at $\xi_{i}(w)$ after applying an automorphism with this prescribed jet at $\xi_{i}(w)$.

For a given point $x$ in a submanifold $M$ of $\mathbb{C}^{n}$ the set $P(x, M)$ of $l$-jets of the form (10) ensuring the osculation up to order $l$ is biholomorphic to a vectorspace and the change of variables in the jet-bundle is affinelinear. This means we have to find a holomorphic section in a locally trivial fibration over $\mathbb{C}^{k}$ of the form

$$
\begin{gathered}
\bigcup_{w \in \mathbb{C}^{k}} P\left(\xi_{i}(w), M(w)\right) \\
\downarrow \\
\mathbb{C}^{k}
\end{gathered}
$$

where the fibers are biholomorphic to a vectorspace $\mathbb{C}^{N}$ and the structure group is $\operatorname{Aff}\left(\mathbb{C}^{N}\right)$, the group of affine linear automorphisms of $\mathbb{C}^{N}$. Since $\mathbb{C}^{N}$ is contractible a continuous section always exists and the OkaGrauert principle (Theorem 7.2) implies the existence of a holomorphic section.

7.5. The proof of Lemma 2.5. To prove Lemma 2.5 we use the following sublemma:

Lemma 7.7. For every point $p=\left(w_{0}, \hat{p}\right) \in K_{M}=K_{1} \times K_{2}$ there is an open neighborhood $V_{p} \times U_{p} \ni p$ in $K_{1} \times M$ and a family of automorphisms $\psi_{t}$ of $\mathbb{C}^{k} \times \mathbb{C}^{n}$ parametrized by $\mathbb{C}^{N}$, where $N=N(m, n, s)=$ $\left(\left(\begin{array}{c}m+s \\ m\end{array}\right)-(m+1)\right)(n-m)$, such that

1. $\psi_{0}=$ Id.

2. Every $\psi_{t}$ satisfies 1. and 2. in Lemma 2.5.

3. There exist an open neighborhood $T$ of 0 in $\mathbb{C}^{N}$ such that

$$
\begin{array}{r}
\Sigma=\left\{t \in T: \text { There exists } p^{\prime} \in V_{p} \times U_{p} \text { such that } \psi_{t}(w, M(w))\right. \\
\text { osculates of order } \left.l \text { in } \psi_{t}\left(p^{\prime}\right)\right\}
\end{array}
$$

is a set of Lebesgue measure zero.

Proof. If $M(w)$ does not osculate of order $l$ in $p$ let $\psi_{t}=$ Id for every $t \in \mathbb{C}^{N}$.

Now suppose that $M(w)$ osculates of order $l$ in $p$. Without loss of generality assume that $p=\left(w_{0}, 0\right)$ and that the tangent plane is given 
by $T_{\left(w_{0}, 0\right)} M(w)=\left\{\left(z_{1}, \ldots, z_{m}, 0, \ldots, 0\right)\right\}$. Let $\pi_{1}: \mathbb{C}^{n} \rightarrow \mathbb{C}^{m}$ denote the projection to the $m$ first coordinates of $\mathbb{C}^{n}$. After a linear change of variables we can assume that $\pi_{1}\left(b_{i}(w)\right) \neq 0 \in \mathbb{C}^{m}$ for $1 \leq i \leq q$ and for $w \in \tilde{V}_{p}$ where $\tilde{V}_{p}$ is some open neighborhood in $K_{1}$.

To control if $\psi(M(w))$ osculates of order $l$ in some point $\psi\left(p^{\prime}\right)$, $p^{\prime} \in \mathbb{C}^{k} \times M(w)$ for a given automorphism $\psi \in \operatorname{Aut}^{\mathrm{k}}\left(\mathbb{C}^{\mathrm{n}}\right)$, consider the map $F^{\psi}: \mathbb{C}^{k} \times M(w) \rightarrow \mathbb{C}^{N}$ where the coordinate functions $F_{\alpha, u}^{\psi}$, enumerated by pairs $(\alpha, u)$, where $\alpha$ is a multi index $\alpha=\left(\alpha_{1}, \alpha_{2}, \ldots, \alpha_{m}\right)$ with $2 \leq|\alpha| \leq l$ and $u \in \mathbb{N}$ satisfies that $m+1 \leq u \leq n$, are given by

$$
F_{\alpha, u}^{\psi}(w, \zeta)=\operatorname{det}\left(\begin{array}{cccc}
\frac{\partial}{\partial \zeta_{1}}(\psi)_{1}(w, \zeta) & \cdots & \frac{\partial}{\partial \zeta_{1}}(\psi)_{m}(w, \zeta) & \frac{\partial}{\partial \zeta_{1}}(\psi)_{u}(w, \zeta) \\
\vdots & \ddots & \vdots & \vdots \\
\frac{\partial}{\partial \zeta_{m}}(\psi)_{1}(w, \zeta) & \cdots & \frac{\partial}{\partial \zeta_{m}}(\psi)_{m}(w, \zeta) & \frac{\partial}{\partial \zeta_{m}}(\psi)_{u}(w, \zeta) \\
\frac{\partial}{\partial \zeta^{\alpha}}(\psi)_{1}(w, \zeta) & \cdots & \frac{\partial}{\partial \zeta^{\alpha}}(\psi)_{m}(w, \zeta) & \frac{\partial}{\partial \zeta^{\alpha}}(\psi)_{u}(w, \zeta)
\end{array}\right)
$$

Here $(\psi)_{i}$ denotes the $i$-th $z$-coordinate function of the map $\psi: \mathbb{C}^{k} \times$ $\mathbb{C}^{n} \rightarrow \mathbb{C}^{k} \times \mathbb{C}^{n}$ and $\left(\zeta_{i}\right)_{1 \leq i \leq m}$ is some fixed system of local coordinates of $M(w)$ near $p$. Then $\psi(M(w))$ osculates of order $l$ in some point $(w, \zeta)$ if and only if $F^{\psi}(w, \zeta)=0$.

If we restrict our attention to a small enough neighborhood $\tilde{U}_{p} \in K_{2}$, containing 0 , we can use $z_{1}, \ldots, z_{m}$ as local coordinates on $M\left(w_{0}\right)$ near $p$, in fact we will use the coordinates $\left(w, z_{1}, \ldots, z_{m}\right)$ in the restriction to $\tilde{V}_{p} \times \tilde{U}_{p}$. To construct the family of automorphisms $\psi_{t}$ of $\mathbb{C}^{k} \times \mathbb{C}^{n}$ we do the following: For every pair $(\alpha, u)$ we choose a holomorphic function $h_{\alpha, u}$ on $\mathbb{C}^{k} \times \mathbb{C}^{m}$ such that

1. $\left(w, h_{\alpha, u}(w, z)\right)=\left(w, z^{\alpha}\right)$ of order at least $l+1$ in $\left(w_{0}, 0\right)$.

2. $\left(w, h_{\alpha, u}\left(w, \pi_{1}\left(b_{i}(w)\right)\right)\right)=(w, 0)$ of order at least $l+1$ for $1 \leq i \leq q$ and for all $w \in \mathbb{C}^{k}$.

3. $\left(w, h_{\alpha, u}\left(w, \pi_{1}\left(a_{i}\right)\right)=(w, 0)\right.$ for $1 \leq i \leq r$ and for all $w \in \mathbb{C}^{k}$.

Now define the map $\psi: \mathbb{C}^{N} \times \mathbb{C}^{k} \times \mathbb{C}^{n} \rightarrow \mathbb{C}^{k} \times \mathbb{C}^{n}$ by

$$
\psi(t, w, z)=\left(w, z+\sum_{(\alpha, u)} t_{(\alpha, u)} h_{\alpha, u}\left(w, z_{1}, \ldots, z_{m}\right) e_{u}\right)
$$

where $e_{u}$ is the $u$-th unit vector, $m+1 \leq u \leq n$.

This construction gives that for every $t \in \overline{\mathbb{C}}^{N}$ the map $\psi_{t}=\psi(t, \cdot)$ is a parametrized automorphism of $\mathbb{C}^{n}$ and because of condition 2 . and 3. $\psi$ fulfills conditions 1 . and 2. of Lemma 2.5. Furthermore $\psi_{0}=\mathrm{Id}$. The only thing left to check is that $\psi$ fulfills condition 3 .. 
Using the fact that $h_{\alpha, u}(w, z)=z^{\alpha}$ we get that

$$
\begin{aligned}
\left.\frac{\partial}{\partial t_{(\alpha, u)}} F_{\alpha, u}^{\psi_{t}}\right|_{\substack{z=0 \\
w_{0}=0}}=-\left.\frac{\partial}{\partial t_{(\alpha, u)}} \sum t_{(\alpha, u)} \frac{\partial}{\partial z^{\alpha}} h_{\alpha, u}(w, z)\right|_{\substack{z=0 \\
w_{0}=0}}= \\
=\left.\frac{\partial}{\partial t_{(\alpha, u)}} \sum t_{(\alpha, u)} \frac{\partial z^{\alpha}}{\partial z^{\alpha}}\right|_{w_{0}=0}=\alpha ! .
\end{aligned}
$$

Moreover the derivative with respect to $t$ depending on other pairs $\left(\alpha^{\prime}, u^{\prime}\right)$ will vanish

$$
\begin{aligned}
\left.\frac{\partial}{\partial t_{\left(\alpha^{\prime}, u^{\prime}\right)}} F_{\alpha, u}^{\psi_{t}}\right|_{\substack{z=0 \\
w_{0}=0}}=-\left.\frac{\partial}{\partial t_{\left(\alpha^{\prime}, u^{\prime}\right)}} \sum t_{(\alpha, u)} \frac{\partial}{\partial z^{\alpha}} h_{\alpha, u}(w, z)\right|_{\substack{z=0 \\
w_{0}=0}}= \\
=\left.\frac{\partial}{\partial t_{\left(\alpha^{\prime}, u^{\prime}\right)}} \sum t_{(\alpha, u)} \frac{\partial z^{\alpha}}{\partial z^{\alpha}}\right|_{\substack{z=0 \\
w_{0}=0}}=0,
\end{aligned}
$$

whenever $u \neq u^{\prime}$ or whenever $u=u^{\prime},\left|\alpha^{\prime}\right| \leq|\alpha|$ and $\alpha^{\prime} \neq \alpha$.

This implies that the map $\Phi: \mathbb{C}^{N} \times \mathbb{C}^{k} \times M \rightarrow \mathbb{C}^{N}$ defined by $\Phi(t, w, z)=F^{\Phi_{t}}(w, z)$ has maximal rank near $\left(0, w_{0}, 0\right)=(0, p)$. Thus there exists an open neighborhood $\Omega_{p}$ of the form $\Omega_{p}=T \times V_{p} \times U_{p}$ of $(0, p)$ in $\mathbb{C}^{N} \times \mathbb{C}^{k} \times M$ such that $\left.\Phi\right|_{\Omega_{p}}$ is transversal to $0 \in \mathbb{C}^{N}$. This implies that for almost all $t \in T$ the map $F^{\psi_{t}}: \mathbb{C}^{k} \times M(w) \rightarrow \mathbb{C}^{N}$ is transversal to 0 . Since $m<N$ this means that for almost all $t \in T$ the image $F^{\psi_{t}}\left(V_{p} \times U_{p}\right)$ does not meet 0, i.e. $\psi_{t}((w, M))$ does not osculate of order $l$ for any $p^{\prime} \in V_{p} \times U_{p}$.

Proof of Lemma 2.5. Choose finitely many open subsets $V_{i} \times U_{i}$ of $\mathbb{C}^{k} \times$ $M$ together with families $\psi: T_{i} \times \mathbb{C}^{k} \times \mathbb{C}^{n} \rightarrow \mathbb{C}^{k} \times \mathbb{C}^{n}$ of automorphisms $i=1,2, \ldots, r$ as in Lemma 7.7 and choose compact subsets $K_{i} \subset V_{i} \times U_{i}$ of $V_{i} \times U_{i}$ which cover $K_{M}$. Since $\psi_{0}^{1}$ is the identity, for $t$ sufficiently small the automorphism $\psi_{t}^{1}$ moves no point of $K_{M}$ more than $\frac{\epsilon}{r}$. So we find $t_{1} \in T_{1}$ such that $\left|P_{2} \phi_{t_{1}}^{1}(w, z)-z\right|<\frac{\epsilon}{r}$ for every $(w, z) \in K$ and the submanifold $\psi_{t_{1}}^{1}\left(\mathbb{C}^{k} \times M\right)$ does not osculate of order $l$ at any point of $\psi_{t_{1}}^{1}\left(K_{1}\right)$.

Observe that the property of not osculating of order $l$ at some point is preserved under small perturbations, i.e. for each compact subset $L$ of a submanifold $M$ of $\mathbb{C}^{n}$ which does not osculate of order $l$ at any point of $L$ there exists some $\epsilon>0$ such that for each automorphism $\psi$ of $\mathbb{C}^{n}$ the property $|\psi(z)-z|<\epsilon$ for every $z \in L$ implies that $\psi(M)$ remains non-osculating of order $l$ at any point of $\psi(L)$ (for holomorphic maps small perturbations in values imply small perturbations in derivatives).

Hence we find a sufficiently small $t_{2} \in T_{2}$ such that first $\mid P_{2} \psi_{t_{2}}^{2}(w, z)-$ $z \mid<\frac{\epsilon}{r}$ for every $z \in \psi^{1}(K)$, second the submanifold $\psi_{t_{2}}^{2} \circ \psi_{t_{1}}^{1}\left(\mathbb{C}^{k} \times M\right)$ does not osculate of order $l$ at any point of $\psi_{t_{2}}^{2} \circ \psi_{t_{1}}^{1}\left(K_{2}\right)$ and third 
$\psi_{t_{2}}^{2} \circ \psi_{t_{1}}^{1}\left(\mathbb{C}^{k} \times M\right)$ remains non-osculating of order $l$ at any point of $\psi_{t_{2}}^{2} \circ \psi_{t_{1}}^{1}\left(K_{1}\right)$. Proceeding by induction we find an automorphism $\psi:=$ $\psi_{t_{r}}^{r} \circ \psi_{t_{r-1}}^{r-1} \circ \cdots \circ \psi_{t_{1}}^{1}$ moving no point of $K$ more than $\epsilon$ and such that $\psi\left(\mathbb{C}^{k} \times M\right)$ does not osculate of order $l$ at any point of $\psi\left(\cup_{i=1}^{r} K_{i}\right) \supset$ $\psi\left(K_{M}\right)$. Since all automorphisms $\psi_{t}$ satisfy properties 1 . and 2., $\psi$ satisfies them as well.

\section{REFERENCES}

[1] E. Andersén, Volume-preserving automorphisms of $\mathbb{C}^{n}$, Complex Variables 14, (1990) p. 223-235

[2] E. Andersén, L. Lempert, On the group of holomorphic automorphisms of $\mathbb{C}^{n}$, Invent. math. 110, (1992) p. 371-388

[3] S. S. Abhyankar, T. T. Moh, Embeddings of the line in the plane, J. Reine Angew. Math. 276 (1975), p. 148-166.

[4] S. Borell, Holomorphic embeddings and Eisenman hyperbolicity, Thesis University of Bern 2007

[5] G. Buzzard, J. E. Fornæss, An embedding of $\mathbb{C}$ into $\mathbb{C}^{2}$ with hyperbolic complement, Math. Ann. 306 (1996), no. 3, p. 539-546.

[6] S. Borell, F. Kutzschebauch, Non-equivalent embeddings into complex euclidean spaces, Intern. J. of Math. 17 (2006), no. 9, p. 1033-1046

[7] S. Bochner, D. Montgomery, Locally compact groups of differentiable transformations, Ann. of Math. (2) 47 (1946), p. 639-653.

[8] H. Derksen, F. Kutzschebauch, Nonlinearizable holomorphic group actions, Math. Ann. 311 (1998), p. 41-53.

[9] H. Derksen, F. Kutzschebauch, Global holomorphic linearization of actions of compact Lie groups on $\mathbb{C}^{n}$, Contemporary Mathematics 222 (1999), p. 201210.

[10] D. A. Eisenman, Intrinsic measures on complex manifolds and holomorphic mappings, Mem. Amer. Math. Soc., no. 96, Amer. Math. Soc., Providence, R.I., 1970.

[11] Y. Eliashberg, M. Gromov, Embeddings of Stein manifolds of dimension $n$ into the affine space of dimension 3n/2 +1, Ann. of Math.(2) 136 (1992), no. 1, p. $123-135$.

[12] O. Forster, Plongements des variétés de Stein, Comm. Math. Helv., 45 (1970), p. $170-184$.

[13] O. Forster and K. J. Ramspott, Analytische Modulgarben und Endromisbündel, Invent. Math. 2 (1966), p. 145-170.

[14] F. Forstnerič, Interpolation by holomorphic automorphisms and embeddings in $\mathbb{C}^{n}$, J. Geom. Anal. 9 (1999), p. 93-117.

[15] F. Forstnerič, The Oka principle for sections of stratified fiber bundles, Preprint, arXiv 0705.0591v4, 2008.

[16] F. Forstnerič, J. Globevnik, J.-P. Rosay, Nonstraightenable complex lines in $\mathbb{C}^{2}$, Ark. Mat. 34 (1996), no. 1, p. 97-101.

[17] F. Forstnerič, B. Ivarsson, F. Kutzschebauch, J. Prezelj, An interpolation theorem for proper holomorphic embeddings, Math. Ann. 338 (2007), no. 3, p. 545554 
[18] F. Forstnerič, J. Prezelj, Oka's principle for holomorphic submersions with sprays, Math. Ann. 322 (2002), p. 633-666.

[19] F. Forstnerič, J-P. Rosay, Approximation of biholomorphic mappings by automorphisms of $\mathbb{C}^{n}$, Invent. math. 112, (1993), p. 323-349

[20] G. Freudenburg, L. Moser-Jauslin, Embeddings of Danielevski surfaces, Math. Z. 245 (2003), p. 823-834.

[21] J. Globevnik, Interpolation by proper holomorphic embeddings of the disc into $\mathbb{C}^{2}$, Math.Res. Lett, 9 (2002), p. 567-577.

[22] I. Graham, H. Wu, Some remarks on the intrinsic measures of Eisenman, Trans. Am. Math. 288 (1985), no. 2, p. 625-660.

[23] H. Grauert, Approximationssätze für holomorphe Funktionen mit Werten in komplexen Räumen, Math. Ann. 133 (1957), 139-159.

[24] H. Grauert, Holomorphe Funktionen mit Werten in komplexen Lieschen Gruppen, Math. Ann. 133 (1957), 450-472.

[25] H. Grauert, Analytische Faserungen über holomorph-vollständigen Räumen, Math. Ann. 135 (1958), 263-273.

[26] M. Gromov, Oka's principle for holomorphic sections of elliptic bundles, J. Amer. Math. Soc. 2 (1989), p. 851-897.

[27] P. Heinzner, F. Kutzschebauch, An equivariant version of Grauert's Oka principle, Invent. math. 119 (1995), p. 317-346

[28] A.T. Huckleberry, Actions of groups of holomorphic transformations, Several complex variables, VI, Encyclopaedia Math. Sci. 69, Springer, Berlin, (1990) p. $143-196$.

[29] B. Ivarsson, F. Kutzschebauch, A solution of Gromov's Vaserstein problem, C.R. Acad. Sci. Paris, 346 (2008), p. 1239-1243

[30] B. Ivarsson, F. Kutzschebauch, Holomorphic factorization of maps into $S L_{n}(\mathbb{C})$, preprint $18 \mathrm{p} ., 2008$

[31] S. Kaliman, Isotopic embeddings of affine algebraic varieties into $\mathbb{C}^{n}$, Contemp. Math. 137 (1992), p. 291-295.

[32] S. Kaliman, F. Kutzschebauch, Criteria for the density property of complex manifolds, Invent. math. 172 (2008), no. 1, p. 71-87

[33] F. Kutzschebauch, Some results on embedding Stein spaces with interpolation, Ark. Mat. 43 (2005), p. 419-425.

[34] F. Kutzschebauch, Compact and reductive subgroups of the group of holomorphic automorphisms of $\mathbb{C}^{n}$, RIMS Kokyuroku 1033 (1998), p. 81-94

[35] F. Kutzschebauch, Andersén-Lempert-theory with parameters: A representation theoretic point of view, J. Algebra Appl. 4, No. 3 (2005), p. 325-340

[36] J. Prezelj, Interpolation of embeddings of Stein manifolds on discrete sets, Math. Ann. 326 (2003), p. 275-296.

[37] R. Remmert, Sur les espaces analytiques holomorphiquement séparables et holomorphiquement convex, C. R. Acad. Sci. Paris 243 (1956), p. 118-121.

[38] J.-P. Rosay, W. Rudin, Holomorphic maps from $\mathbb{C}^{n}$ to $\mathbb{C}^{n}$, Trans. Amer. Math. Soc. 310 (1988), no. 1, p. 47-86.

[39] J.-P. Rosay, W. Rudin, Holomorphic embeddings of $\mathbb{C}$ in $\mathbb{C}^{n}$, in: Several Complex Variables, Math. Notes 38, Proceedings of the Mittag-Leffler Institute 1987-88, Princeton University Press (1993), p. 563-569.

[40] J. Schürmann, Embeddings of Stein spaces into affine spaces of minimal dimension, Math. Ann. 307 (1997), no. 3, p. 381-399. 
[41] M. Suzuki, Sur les opérationes holomorphes du group additif complexe sur l'espace de deux variables complexes, Ann. scient. Ec. Norm. Sup. 4 série 10 (1977), p. 517-546

[42] D. Varolin, The density property for complex manifolds and geometric structures J. Geom. Anal. 11 (2001), no. 1, p. 135-160.

[43] E. Fornæss Wold, Fatou-Bieberbach domains, Intern. J. of Math. 16 no. 10 (2005), p. 1119-1130

[44] M. Zaŭdenberg, An analytic cancellation theorem and exotic algebraic structures on $C^{n}, n \geq 3$, Colloque d'Analyse Complexe et Géométrie (Marseille, 1992), Astérisque 8 (1993), no. 217, p. 251-282.

Institute of Mathematics, University of Bern, Sidlerstrasse 5, CH3012 BERN, SWITZERLAND

E-mail address: frank.kutzschebauch@math.unibe.ch

Dept. of Natural Sciences, Engineering and Mathematics, Mid SweDen University, SE-851 70 Sundsvall, Sweden

E-mail address: sam.lodin@miun.se 